\title{
Genetic Mapping of Two Blood Pressure Quantitative Trait Loci on Rat Chromosome 1
}

\author{
Lingping Gu, ${ }^{\star}$ Howard Dene, ${ }^{\star}$ Alan Y. Deng, ${ }^{\star}$ Barbara Hoebee, ${ }^{\ddagger}$ Marie-Therese Bihoreau, ${ }^{\S}$ Michael James, ${ }^{\S}$ and John P. Rapp ${ }^{\star}$ \\ *Department of Physiology and Molecular Medicine, Medical College of Ohio, Toledo, Ohio 43699; ${ }^{\ddagger}$ Laboratory of Carcinogenesis and \\ Mutagenesis, National Institute of Public Health and Environmental Protection, Bilthoven 3720 BA, The Netherlands; and ${ }^{\S}$ Wellcome \\ Trust Centre for Human Genetics, University of Oxford, Oxford OX3 7BN, England
}

\begin{abstract}
A genetic map for rat chromosome 1 was constructed using 66 microsatellite markers typed on either or both of two populations derived from inbred Dahl salt-sensitive $(\mathrm{S})$ rats: $\mathrm{F}_{2}(\mathrm{LEW} \times \mathrm{S}) n=151$, and $\mathrm{F}_{2}(\mathrm{WKY} \times \mathrm{S}) n=159$. These populations had been raised on a high salt $(8 \% \mathrm{NaCl})$ diet. Systolic blood pressure and heart weight were found to be genetically linked to two separate regions on rat chromosome 1 in the $\mathrm{F}_{2}(\mathrm{LEW} \times \mathrm{S})$ population. One region was centered around the anonymous SA locus and accounted for 24 $\mathrm{mmHg}$ of blood pressure. The other region was $55 \mathrm{cM}$ from the SA locus centered around a cluster of cytochromes P450 loci, and accounted for $30 \mathrm{mmHg}$ of blood pressure. Since blood pressure and heart weight were highly correlated these same regions were also linked to heart weight. These results were cross-specific as linkage of these chromosome 1 regions to blood pressure and heart weight was not observed in several other $F_{2}$ populations derived by crossing $S$ and other normotensive control strains. This is presumably due to different alleles and/or different genetic backgrounds in the various populations. The $\mathrm{SA}$ region of chromosome 1 was also found to influence body weight in $\mathrm{F}_{2}(\mathrm{LEW} \times \mathrm{S})$ rats. Combining the present data with our previously published data on the $\mathrm{F}_{2}(\mathrm{LEW} \times \mathrm{S})$ population showed that four separate quantitative trait loci with additive effects accounted for $106 \mathrm{mmHg}$ and $38 \%$ of the total variance of blood pressure and for $506 \mathrm{mg}$ and $34 \%$ of the total variance of heart wt. (J. Clin. Invest. 1996. 97:777-788.) Key words: Dahl rats - hypertension - body weight - SA locus - cytochrome P450
\end{abstract}

\section{Introduction}

Identification of the multiple genetic loci controlling continuously varying (quantitative) genetic traits such as blood pressure, is a major challenge. Using many genetic markers and linkage analysis it is possible to identify chromosomal regions that contain quantitative trait loci $(\mathrm{QTL})^{1}(1,2)$. The identification of the actual QTL, as opposed to the chromosomal region containing the QTL, remains a more formidable obstacle

Address correspondence to John Rapp, Department of Physiology \& Molecular Medicine, Medical College of Ohio, P.O. Box 10008, Toledo, OH 43699-0008. Phone: 419-381-4291; FAX: 419-381-3124.

Received for publication 5 September 1995 and accepted in revised form 16 November 1995.

J. Clin. Invest.

(c) The American Society for Clinical Investigation, Inc.

0021-9738/96/02/0777/12 \$2.00

Volume 97, Number 3, February 1996, 777-788
$(3,4)$. This paper deals with the identification of chromosomal regions influencing blood pressure using an animal model of hypertension, the Dahl salt-sensitive (S) rat. This hypertensive strain and others have been used previously to locate such regions (4).

Among the loci known to cosegregate with blood pressure is a gene of unknown function called the $S A$ gene. This gene was originally cloned by Iwai and Inagami (5) based on its greater expression in the kidneys of spontaneously hypertensive rats (SHR) compared to Wistar-Kyoto (WKY) normotensive controls. The $S A$ gene has been linked to markers on rat chromosome $1(6,7)$. Alleles at the $S A$ locus were shown to cosegregate with blood pressure in $\mathrm{F}_{2}$ populations derived from an SHR $\times$ WKY cross $(8,9)$, a stroke prone-SHR $\times$ WKY cross (6), and an $\mathrm{F}_{2}$ population derived from an $\mathrm{S} \times$ Lewis cross (10). The $S A$ locus in humans was linked to blood pressure in a Japanese population (11) but not in a European population (12).

In an attempt to improve the genetic map of rat chromosome 1, and to determine if the blood pressure effect in fact localizes maximally at or very close to the $S A$ locus, we developed an improved genetic map for chromosome 1 . We have found that rat chromosome 1 contains two blood pressure QTL, one very close or identical to the $S A$ locus and a second QTL about 55 centiMorgans (cM) from the $S A$ locus.

\section{Methods}

Animal procedure. Inbred Dahl salt-sensitive (SS/Jr) and inbred Dahl salt-resistant (SR/Jr) rat strains (13) were from our colony at Medical College of Ohio and will be referred to hereafter as $\mathrm{S}$ and $\mathrm{R}$ rats. Lewis rats (LEW/NCrLBR) were obtained from Charles River Laboratories (Wilmington, MA) and are referred to as LEW. Spontaneously hypertensive rats (SHR/NHsd), Wistar-Kyoto (WKY/NHsd) and Brown Norway (BN/SsNHsd) rats were obtained from Harlan Sprague-Dawley (Indianapolis, IN) and will be referred to as SHR, WKY, and BN, respectively. The Milan normotensive strain (MNS) was obtained from the Veterinary Resources Branch of the National Institutes of Health (Bethesda, MD). Albino surgery (AS) rats were obtained from C. Heatherington (National Institute for Medical Research, The Ridgeway, Mill Hill, UK).

Five large $F_{2}$ populations of male rats were used for blood pressure cosegregation analysis and have been described in previous work (14). Briefly, the $F_{2}$ populations were obtained by crossing $S$ males with various contrasting strain (LEW, WKY, R, BN, and MNS) females, and then intercrossing the $F_{1}$ progeny to produce $F_{2}$ rats. The

1. Abbreviations used in this paper: AS, Albino surgery rat; BN, brown Norway rat; DOP, degenerate oligonucleotide primer; LEW, Lewis rat; MNS, Milan normotensive strain; QTL, quantitative trait locus; R, inbred Dahl salt-resistant rat; S, inbred Dahl salt-sensitive rat; SHR, spontaneously hypertensive rat; WKY, Wistar-Kyoto rat. 
Table I. Microsatellite PCR Primers and Rat Strain Variation

\begin{tabular}{|c|c|c|c|c|}
\hline Locus name & Gene name & $\begin{array}{c}\text { GenBank } \\
\text { accession number }\end{array}$ & Primers $\left(5^{\prime}-3^{\prime}\right)$ & $\begin{array}{l}\text { Length variation of the PCR-product } \\
\text { among } 8 \text { inbred strains }\end{array}$ \\
\hline$A L D O A$ & Aldolase A & X04264 & $\begin{array}{l}\text { CAGCCCACTGCCAATAAACAGC } \\
\text { ССТАСССТTСТСАAАGСТСТGC }\end{array}$ & $\mathrm{LEW}<\mathrm{BN}<\mathrm{WKY}<\mathrm{S}=\mathrm{R}=\mathrm{SHR}<\mathrm{MNS}=\mathrm{AS}$ \\
\hline$A T P 1 A 3$ & $\begin{array}{l}\text { ATPase alpha } 3 \\
\text { subunit }\end{array}$ & M90659 & $\begin{array}{l}\text { TGAGCTTCTGGTTGAAGGATCG } \\
\text { CTCCACATATACCACCAAAGGC }\end{array}$ & $\mathrm{BN}=\mathrm{WKY}<\mathrm{SHR}<\mathrm{S}=\mathrm{R}=\mathrm{LEW}=\mathrm{MNS}=\mathrm{AS}$ \\
\hline ATP4A & $\begin{array}{l}\text { Gastric } \\
\text { H, K-ATPase }\end{array}$ & M63963 & $\begin{array}{l}\text { CTCTTCTTGATTGGTGGAAC } \\
\text { ATGAGGCTTCGACTTACC }\end{array}$ & $\mathrm{S}=\mathrm{R}=\mathrm{BN}=\mathrm{LEW}=\mathrm{AS}<\mathrm{WKY}=\mathrm{SHR}=\mathrm{MNS}$ \\
\hline$C A L M 3$ & Calmodulin III & X14265 & $\begin{array}{l}\text { CCACAACCCTCTCTACTTGC } \\
\text { TATGCCGAAAGGATTTATGGCG }\end{array}$ & $\mathrm{BN}=\mathrm{LEW}=\mathrm{AS}<\mathrm{S}=\mathrm{R}=\mathrm{MNS}<\mathrm{WKY}=\mathrm{SHR}$ \\
\hline$C E A R$ & $\begin{array}{c}\text { Carcinoembryonic } \\
\text { antigen-related } \\
\text { protein (CGM4) }\end{array}$ & $\begin{array}{l}\text { M32475 } \\
\text { J05417 }\end{array}$ & $\begin{array}{l}\text { GCTCATCATCATCATCATCACC } \\
\text { CCССТGCTTCTTCTTATTTACC }\end{array}$ & $\mathrm{BN}=\mathrm{LEW}=\mathrm{AS}<\mathrm{WKY}=\mathrm{SHR}<\mathrm{S}=\mathrm{R}=\mathrm{MNS}$ \\
\hline$C Y P E$ & Cytochrome P450e & Y00410 & $\begin{array}{l}\text { AGGAAAAGCATATAGAACACGC } \\
\text { TTTACTTACTAGGGCATGGGAT }\end{array}$ & $\mathrm{S}<\mathrm{MNS}<\mathrm{WKY}=\mathrm{SHR}<\mathrm{R}=\mathrm{BN}=\mathrm{LEW}=\mathrm{AS}$ \\
\hline$C Y P 2 A 1$ & $\begin{array}{l}\text { Cytochrome P450 } \\
\text { IIA1 }\end{array}$ & M33312 & $\begin{array}{l}\text { AAGAGAGAAACACTCTGTGG } \\
\text { TTCCAGTAGGAGACTAAAGTGG }\end{array}$ & $\mathrm{S}=\mathrm{WKY}=\mathrm{SHR}=\mathrm{MNS}<\mathrm{R}=\mathrm{BN}=\mathrm{LEW}=\mathrm{AS}$ \\
\hline$C Y P 2 A 2$ & $\begin{array}{l}\text { Cytochrome P450 } \\
\text { IIA2 }\end{array}$ & M33313 & $\begin{array}{l}\text { TATAAAAGCCCCATGCTGGAGC } \\
\text { GGCAAATAGGAAGAAAGGTGGC }\end{array}$ & $\mathrm{WYK}=\mathrm{SHR}=\mathrm{MNS}<\mathrm{S}<\mathrm{R}=\mathrm{BN}=\mathrm{LEW}<\mathrm{AS}$ \\
\hline$C Y P 2 A 3$ & $\begin{array}{l}\text { Cytochrome P450 } \\
\text { IIA3 }\end{array}$ & M33190 & $\begin{array}{l}\text { TACACATGCACCTGCATGCA } \\
\text { TCTTGCCAGTTGCTAAAAATGAG }\end{array}$ & $\mathrm{S}=\mathrm{MNS}<\mathrm{WKY}=\mathrm{SHR}<\mathrm{R}=\mathrm{BN}=\mathrm{LEW}=\mathrm{AS}$ \\
\hline$C Y P 2 B 2$ & $\begin{array}{l}\text { Cytochome P450 } \\
\text { 2B2 }\end{array}$ & S51970 & $\begin{array}{l}\text { TGGCTTGGAGCAGAAAAGTTGG } \\
\text { СТTCTTGAAGGTTGTGTCCACG }\end{array}$ & $\mathrm{S}=\mathrm{BN}<\mathrm{AS}<\mathrm{R}=\mathrm{LEW}=\mathrm{MNS}<\mathrm{WKY}=\mathrm{SHR}$ \\
\hline CYP2C12 & $\begin{array}{l}\text { Cytochrome P450 } \\
\text { 15-beta gene }\end{array}$ & M33545 & $\begin{array}{l}\text { GAAAAGGCTACTAAAGGACTGG } \\
\text { ATAGAGTGCCAACTATGCC }\end{array}$ & $\mathrm{BN}<\mathrm{WKY}=\mathrm{SHR}<\mathrm{S}=\mathrm{R}=\mathrm{MNS}<\mathrm{LEW}=\mathrm{AS}$ \\
\hline D1Mco1 & $\begin{array}{l}\text { Random cloned } \\
\text { SSR }\end{array}$ & U19349 & $\begin{array}{l}\text { CTTATCAGCGAATGGATAC } \\
\text { CСTATTTGTATTTCATCCC }\end{array}$ & $\mathrm{AS}<\mathrm{LEW}=\mathrm{BN}=\mathrm{WKY}=\mathrm{SHR}=\mathrm{MNS}<\mathrm{S}=\mathrm{R}$ \\
\hline $\mathrm{D} 1 \mathrm{Mco} 2$ & $\begin{array}{l}\text { Random cloned } \\
\text { SSR }\end{array}$ & U19350 & $\begin{array}{l}\text { ATGTGGTAAGAGTTTGAATC } \\
\text { GAAATAATAGAGTATGTGTGGG }\end{array}$ & $\mathrm{LEW}=\mathrm{MNS}<\mathrm{S}=\mathrm{R}<\mathrm{BN}<\mathrm{WKY}=\mathrm{SHR}=\mathrm{AS}$ \\
\hline $\mathrm{D} 1 \mathrm{Mco} 3$ & $\begin{array}{l}\text { Random cloned } \\
\text { SSR }\end{array}$ & U19351 & $\begin{array}{l}\text { GTAAAGTGGTGTCTGTGCC } \\
\text { TTGCATGAAATGATGTGTG }\end{array}$ & $\mathrm{S}<\mathrm{R}=\mathrm{LEW}=\mathrm{BN}=\mathrm{MNS}=\mathrm{AS}<\mathrm{WKY}=\mathrm{SHR}$ \\
\hline D1Mco4 & $\begin{array}{l}\text { Random cloned } \\
\text { SSR }\end{array}$ & U19352 & $\begin{array}{l}\text { CGGTGTCACAAGATTTGTC } \\
\text { GAGTATGATACCCAGGACTC }\end{array}$ & $\mathrm{S}=\mathrm{R}=\mathrm{WKY}=\mathrm{MNS}=\mathrm{AS}<\mathrm{LEW}=\mathrm{BN}=\mathrm{SHR}$ \\
\hline $\mathrm{D} 1 \mathrm{Mco} 5$ & $\begin{array}{l}\text { Random cloned } \\
\text { SSR }\end{array}$ & U19357 & $\begin{array}{l}\text { ACGGTCACTGGGTTCTTG } \\
\text { TCACTAGCTTCCTAGGGC }\end{array}$ & $\mathrm{WKY}=\mathrm{SHR}<\mathrm{LEW}<\mathrm{S}=\mathrm{R}=\mathrm{BN}=\mathrm{AS}<\mathrm{MNS}$ \\
\hline D1Mco6 & $\begin{array}{l}\text { Random cloned } \\
\text { SSR }\end{array}$ & U29197 & $\begin{array}{l}\text { CTTCAGTGAGAGAAGATGCACC } \\
\text { CCAACATTTCСТCATCCC }\end{array}$ & $\mathrm{WKKY}<\mathrm{LEW}=\mathrm{BN}=\mathrm{SHR}=\mathrm{AS}<\mathrm{S}=\mathrm{R}$ \\
\hline D0Mco1* & $\begin{array}{l}\text { Random cloned } \\
\text { SSR }\end{array}$ & U19353 & $\begin{array}{l}\text { CTTTATGTGGGTTCTGAGG } \\
\text { AGGTATTGAGATGTAGGATATG }\end{array}$ & $\mathrm{S}=\mathrm{R}=\mathrm{LEW}=\mathrm{BN}=\mathrm{WKY}=\mathrm{SHR}=\mathrm{MNS}=\mathrm{AS}$ \\
\hline D0Mco $2^{\ddagger}$ & $\begin{array}{l}\text { Random cloned } \\
\text { SSR }\end{array}$ & U19354 & $\begin{array}{l}\text { CAGCCTTCATTCTCACAC } \\
\text { TGACTTCTGTGAGCTCCTAC }\end{array}$ & $\mathrm{MNS}<\mathrm{WKY}=\mathrm{SHR}=\mathrm{AS}<\mathrm{S}=\mathrm{R}=\mathrm{LEW}<\mathrm{BN}$ \\
\hline D0Mco3* & $\begin{array}{l}\text { Random cloned } \\
\text { SSR }\end{array}$ & U19355 & $\begin{array}{l}\text { CTCAGAGACAAAGCTAAGG } \\
\text { AGGAAACATACAGACCCAG }\end{array}$ & $\mathrm{S}=\mathrm{R}=\mathrm{LEW}=\mathrm{WKY}=\mathrm{NB}=\mathrm{SHR}=\mathrm{MNS}=\mathrm{AS}$ \\
\hline D0Mco $4^{\ddagger}$ & $\begin{array}{l}\text { Random cloned } \\
\text { SSR }\end{array}$ & U19356 & $\begin{array}{l}\text { GGCACGTGTACATACTGATG } \\
\text { TCTATTCAGGCCTCTGAC }\end{array}$ & $\mathrm{LEW}=\mathrm{BN}<\mathrm{S}=\mathrm{R}=\mathrm{WKY}=\mathrm{SHR}=\mathrm{MNS}=\mathrm{AS}$ \\
\hline D1Mgh1* & $\begin{array}{l}\text { Random cloned } \\
\text { SSR }\end{array}$ & & Research Genetics & $\mathrm{BN}<\mathrm{SHR}<\mathrm{S}=\mathrm{R}=\mathrm{LEW}=\mathrm{WKY}<\mathrm{MNS}=\mathrm{AS}$ \\
\hline D1Mgh2 & $\begin{array}{l}\text { Random cloned } \\
\text { SSR }\end{array}$ & & Research Genetics & $\mathrm{WKY}=\mathrm{SHR}<\mathrm{S}=\mathrm{R}=\mathrm{LEW}=\mathrm{BN}=\mathrm{MNS}=\mathrm{AS}$ \\
\hline D1Mgh3 & $\begin{array}{l}\text { Random cloned } \\
\text { SSR }\end{array}$ & & Research Genetics & $\mathrm{BN}<\mathrm{WKY}=\mathrm{SHR}<\mathrm{S}=\mathrm{R}=\mathrm{MNS}=\mathrm{AS}<\mathrm{LEW}$ \\
\hline D1Mgh4 & $\begin{array}{l}\text { Random cloned } \\
\text { SSR }\end{array}$ & & Research Genetics & $\mathrm{LEW}=\mathrm{BN}=\mathrm{MNS}=\mathrm{AS}<\mathrm{S}=\mathrm{R}<\mathrm{WKY}=\mathrm{SHR}$ \\
\hline D1Mgh6 & $\begin{array}{l}\text { Random cloned } \\
\text { SSR }\end{array}$ & & Research Genetics & $\mathrm{S}=\mathrm{R}=\mathrm{LEW}=\mathrm{BN}=\mathrm{MNS}=\mathrm{AS}<\mathrm{WKY}=\mathrm{SHR}$ \\
\hline D1Mgh7 & $\begin{array}{l}\text { Random cloned } \\
\text { SSR }\end{array}$ & & Research Genetics & $\mathrm{LEW}=\mathrm{BN}<\mathrm{MNS}<\mathrm{WKY}=\mathrm{SHR}<\mathrm{S}=\mathrm{R}=\mathrm{AS}$ \\
\hline
\end{tabular}


Table I. Continued

\begin{tabular}{|c|c|c|c|c|}
\hline Locus name & Gene name & $\begin{array}{c}\text { GenBank } \\
\text { accession number }\end{array}$ & Primers $\left(5^{\prime}-3^{\prime}\right)$ & $\begin{array}{l}\text { Length variation of the PCR-product } \\
\text { among } 8 \text { inbred strains }\end{array}$ \\
\hline D1Mgh8 & $\begin{array}{l}\text { Random cloned } \\
\text { SSR }\end{array}$ & & Research Genetics & $\mathrm{LEW}<\mathrm{S}=\mathrm{R}=\mathrm{MNS}<\mathrm{BN}<\mathrm{WKY}=\mathrm{SHR}<\mathrm{AS}$ \\
\hline D1Mgh9 & $\begin{array}{l}\text { Random cloned } \\
\text { SSR }\end{array}$ & & Research Genetics & $\mathrm{LEW}=\mathrm{MNS}<\mathrm{S}=\mathrm{R}=\mathrm{WIY}=\mathrm{SHR}=\mathrm{AS}<\mathrm{BN}$ \\
\hline D1Mgh10 & $\begin{array}{l}\text { Random cloned } \\
\text { SSR }\end{array}$ & & Research Genetics & $\mathrm{S}=\mathrm{R}=\mathrm{LEW}=\mathrm{BN}=\mathrm{AS}<\mathrm{MNS}<\mathrm{WKY}=\mathrm{SHR}$ \\
\hline D1Mgh11 & $\begin{array}{l}\text { Random cloned } \\
\text { SSR }\end{array}$ & & Research Genetics & $\mathrm{WKY}=\mathrm{SHR}<\mathrm{S}=\mathrm{LEW}=\mathrm{BN}=\mathrm{AS}<\mathrm{MNS}<\mathrm{R}$ \\
\hline D1Mgh12 & $\begin{array}{l}\text { Random cloned } \\
\text { SSR }\end{array}$ & & Research Genetics & $\mathrm{LEW}=\mathrm{AS}<\mathrm{S}=\mathrm{R}<\mathrm{BN}=\mathrm{MNS}<\mathrm{WKY}=\mathrm{SHR}$ \\
\hline D1Mgh13 & $\begin{array}{l}\text { Random cloned } \\
\text { SSR }\end{array}$ & & Research Genetics & $\mathrm{BN}=\mathrm{MNS}<\mathrm{AS}<\mathrm{S}=\mathrm{R}=\mathrm{LEW}<\mathrm{WKY}<\mathrm{SHR}$ \\
\hline D1Mgh14 & $\begin{array}{l}\text { Random cloned } \\
\text { SSR }\end{array}$ & & Research Genetics & $\mathrm{BN}<\mathrm{S}=\mathrm{SHR}<\mathrm{R}=\mathrm{LEW}=\mathrm{WKY}=\mathrm{MNS}=\mathrm{AS}$ \\
\hline D1Mgh15* & $\begin{array}{l}\text { Random cloned } \\
\text { SSR }\end{array}$ & & Research Genetics & $\mathrm{S}=\mathrm{R}=\mathrm{LEW}=\mathrm{WKY}=\mathrm{SHR}=\mathrm{MNS}=\mathrm{AS}<\mathrm{BN}$ \\
\hline D1Mgh16 & $\begin{array}{l}\text { Random cloned } \\
\text { SSR }\end{array}$ & & Research Genetics & $\mathrm{LEW}=\mathrm{WKY}=\mathrm{SHR}=\mathrm{MNS}<\mathrm{S}=\mathrm{R}=\mathrm{AS}<\mathrm{BN}$ \\
\hline D1Mgh17* & $\begin{array}{l}\text { Random cloned } \\
\text { SSR }\end{array}$ & & Research Genetics & $\mathrm{S}=\mathrm{R}=\mathrm{LEW}=\mathrm{BN}=\mathrm{WKY}=\mathrm{SHR}=\mathrm{MNS}=\mathrm{AS}$ \\
\hline D1Mgh1 $18^{\ddagger}$ & $\begin{array}{l}\text { Random cloned } \\
\text { SSR }\end{array}$ & & Research Genetics & $\mathrm{R}<\mathrm{WKY}<\mathrm{SHR}<\mathrm{S}=\mathrm{LEW}=\mathrm{BN}=\mathrm{MNS}=\mathrm{AS}$ \\
\hline D1Mgh19 & $\begin{array}{l}\text { Random cloned } \\
\text { SSR }\end{array}$ & & Research Genetics & $\mathrm{WKY}=\mathrm{SHR}<\mathrm{S}=\mathrm{R}=\mathrm{BN}=\mathrm{AS}<\mathrm{LEW}=\mathrm{MNS}$ \\
\hline $\mathrm{D} 1 \mathrm{Mgh} 20$ & $\begin{array}{l}\text { Random cloned } \\
\text { SSR }\end{array}$ & & Research Genetics & $\mathrm{BN}<\mathrm{AS}<\mathrm{S}=\mathrm{R}=\mathrm{WKY}=\mathrm{SHR}<\mathrm{MNS}<\mathrm{LEW}$ \\
\hline $\mathrm{D} 1 \mathrm{Mgh} 21$ & $\begin{array}{l}\text { Random cloned } \\
\text { SSR }\end{array}$ & & Research Genetics & $\mathrm{LEW}<\mathrm{S}=\mathrm{R}=\mathrm{WKY}<\mathrm{SHR}=\mathrm{MNS}<\mathrm{BN}=\mathrm{AS}$ \\
\hline D1Mit2* & $\begin{array}{l}\text { Random cloned } \\
\text { SSR }\end{array}$ & & Research Genetics & $\mathrm{S}=\mathrm{LEW}=\mathrm{WKY}=\mathrm{AS}<\mathrm{SHR}=\mathrm{MNS}<\mathrm{BN}<\mathrm{R}$ \\
\hline D1Mit3 & $\begin{array}{l}\text { Random cloned } \\
\text { SSR }\end{array}$ & & Research Genetics & $\mathrm{SHR}=\mathrm{MNS}<\mathrm{S}=\mathrm{R}=\mathrm{LEW}<\mathrm{WKY}=\mathrm{AS}<\mathrm{BN}$ \\
\hline D1Mit4 & $\begin{array}{l}\text { Random cloned } \\
\text { SSR }\end{array}$ & & Research Genetics & $\mathrm{S}=\mathrm{R}=\mathrm{BN}<\mathrm{LEW}=\mathrm{MNS}<\mathrm{WKY}=\mathrm{SHR}=\mathrm{AS}$ \\
\hline D1Mit5 & $\begin{array}{l}\text { Random cloned } \\
\text { SSR }\end{array}$ & & Research Genetics & $\mathrm{LEW}=\mathrm{BN}<\mathrm{WKY}=\mathrm{SHR}<\mathrm{S}=\mathrm{R}=\mathrm{MNS}=\mathrm{AS}$ \\
\hline D1Mit7 & $\begin{array}{l}\text { Random cloned } \\
\text { SSR }\end{array}$ & & Research Genetics & $\mathrm{SHR}<\mathrm{WKY}=\mathrm{AS}<\mathrm{S}=\mathrm{R}=\mathrm{LEW}=\mathrm{MNS}<\mathrm{BN}$ \\
\hline D1Mit8 & $\begin{array}{l}\text { Random cloned } \\
\text { SSR }\end{array}$ & & Research Genetics & $\mathrm{WKY}=\mathrm{SHR}=\mathrm{MNS}=\mathrm{AS}<\mathrm{LEW}=\mathrm{BN}<\mathrm{S}=\mathrm{R}$ \\
\hline D1Mit9 & $\begin{array}{l}\text { Random cloned } \\
\text { SSR }\end{array}$ & & Research Genetics & $\mathrm{WKY}=\mathrm{SHR}<\mathrm{MNS}<\mathrm{S}=\mathrm{R}<\mathrm{LEW}=\mathrm{BN}=\mathrm{AS}$ \\
\hline D1Mit10* & $\begin{array}{l}\text { Random cloned } \\
\text { SSR }\end{array}$ & & Research Genetics & $\mathrm{BN}<\mathrm{S}=\mathrm{R}=\mathrm{LEW}=\mathrm{WKY}=\mathrm{SHR}=\mathrm{MNS}=\mathrm{AS}$ \\
\hline D1Mit12 & $\begin{array}{l}\text { Random cloned } \\
\text { SSR }\end{array}$ & & Research Genetics & $\mathrm{AS}<\mathrm{BN}<\mathrm{S}=\mathrm{R}<\mathrm{WKY}=\mathrm{SHR}<\mathrm{LEW}=\mathrm{MNS}$ \\
\hline D1Mit14 & $\begin{array}{l}\text { Random cloned } \\
\text { SSR }\end{array}$ & & Research Genetics & $\mathrm{S}=\mathrm{R}=\mathrm{SHR}<\mathrm{LEW}=\mathrm{WKY}<\mathrm{MNS}<\mathrm{AS}<\mathrm{BN}$ \\
\hline D1Mit15* & $\begin{array}{l}\text { Random cloned } \\
\text { SSR }\end{array}$ & & Research Genetics & $\mathrm{B}<\mathrm{S}=\mathrm{R}=\mathrm{LEW}=\mathrm{WKY}=\mathrm{SHR}=\mathrm{AS}<\mathrm{MNS}$ \\
\hline D1N40 & $\begin{array}{l}\text { DNA segment, } \\
\text { STMS }\end{array}$ & L08080 & $\begin{array}{l}\text { TGACCTAGCATGAGCAGG } \\
\text { GCCCAACTTGCTTTTTGG }\end{array}$ & $\mathrm{WKY}=\mathrm{SHR}<\mathrm{S}=\mathrm{R}=\mathrm{BN}=\mathrm{LEW}=\mathrm{MNS}=\mathrm{AS}$ \\
\hline D1N64 & $\begin{array}{l}\text { DNA segment, } \\
\text { STMS }\end{array}$ & L08081 & $\begin{array}{l}\text { ATGTGTTTGTGGGTGGTTCCAGCAGG } \\
\text { тCTCTCTCTCTCTCTCGTGTGTGT }\end{array}$ & $\mathrm{LEW}=\mathrm{WKY}=\mathrm{SHR}=\mathrm{MNS}=\mathrm{AS}<\mathrm{S}=\mathrm{R}<\mathrm{BN}$ \\
\hline D7Mit45 & $\begin{array}{l}\text { Random cloned } \\
\text { SSR }\end{array}$ & & Research Genetics & $\mathrm{BN}=\mathrm{LEW}<\mathrm{WKY}=\mathrm{SHR}<\mathrm{S}=\mathrm{R}=\mathrm{MNS}=\mathrm{AS}$ \\
\hline
\end{tabular}


Table I. Continued

\begin{tabular}{|c|c|c|c|c|}
\hline Locus name & Gene name & $\begin{array}{c}\text { GenBank } \\
\text { accession number }\end{array}$ & Primers $\left(5^{\prime}-3^{\prime}\right)$ & $\begin{array}{l}\text { Length variation of the PCR-product } \\
\text { among } 8 \text { inbred strains }\end{array}$ \\
\hline D7Mit62 & $\begin{array}{l}\text { Random cloned } \\
\text { SSR }\end{array}$ & & Research Genetics & $\mathrm{S}=\mathrm{R}=\mathrm{WKY}=\mathrm{SHR}=\mathrm{AS}<\mathrm{LEW}=\mathrm{BN}=\mathrm{MNS}$ \\
\hline D7Mit66 & $\begin{array}{l}\text { Random cloned } \\
\text { SSR }\end{array}$ & & Research Genetics & $\mathrm{BN}<\mathrm{S}=\mathrm{R}=\mathrm{WKY}=\mathrm{AS}<\mathrm{MNS}<\mathrm{LEW}=\mathrm{SHR}$ \\
\hline D7Mit69 & $\begin{array}{l}\text { Random cloned } \\
\text { SSR }\end{array}$ & & Research Genetics & $\mathrm{S}=\mathrm{R}=\mathrm{AS}<\mathrm{BN}=\mathrm{LEW}=\mathrm{WKY}=\mathrm{SHR}=\mathrm{MNS}$ \\
\hline D7Mit87 & $\begin{array}{l}\text { Random cloned } \\
\text { SSR }\end{array}$ & & Research Genetics & $\mathrm{S}=\mathrm{R}=\mathrm{AS}=\mathrm{SHR}=\mathrm{MNS}<\mathrm{BN}=\mathrm{LEW}=\mathrm{WKY}$ \\
\hline D7Mit89 & $\begin{array}{l}\text { Random cloned } \\
\text { SSR }\end{array}$ & & Research Genetics & $\mathrm{LEW}=\mathrm{MNS}=\mathrm{AS}<\mathrm{S}=\mathrm{R}=\mathrm{BN}=\mathrm{WKY}=\mathrm{SHR}$ \\
\hline D7Mit99 & $\begin{array}{l}\text { Random Cloned } \\
\text { SSR }\end{array}$ & & Research Genetics & $\mathrm{WKY}<\mathrm{S}=\mathrm{R}=\mathrm{BN}=\mathrm{LEW}=\mathrm{SHR}=\mathrm{MNS}=\mathrm{AS}$ \\
\hline D7Mit101 & $\begin{array}{l}\text { Random Cloned } \\
\text { SSR }\end{array}$ & & Research Genetics & $\mathrm{S}=\mathrm{R}=\mathrm{WKY}=\mathrm{SHR}=\mathrm{MNS}<\mathrm{LEW}=\mathrm{AS}<\mathrm{BN}$ \\
\hline D7Mit109 & $\begin{array}{l}\text { Random Cloned } \\
\text { SSR }\end{array}$ & & Research Genetics & $\mathrm{WKY}=\mathrm{SHR}<\mathrm{S}=\mathrm{R}=\mathrm{MNS}=\mathrm{AS}<\mathrm{LEW}<\mathrm{BN}$ \\
\hline $\mathrm{EN} 3 \mathrm{C}^{\S}$ & & & Research Genetics & $\mathrm{SHR}=\mathrm{MNS}<\mathrm{BN}<\mathrm{S}=\mathrm{R}=\mathrm{AS}<\mathrm{LEW}=\mathrm{WKY}$ \\
\hline$I G F 2$ & $\begin{array}{l}\text { Insulin-like } \\
\text { growth factor }\end{array}$ & X17012 & $\begin{array}{l}\text { GATTATACCCACACGTACATGC } \\
\text { AAACCATGCAAACTGCTCAGGG }\end{array}$ & $\mathrm{BN}=\mathrm{LEW}<\mathrm{S}=\mathrm{R}<\mathrm{WKY}=\mathrm{SHR}=\mathrm{AS}<\mathrm{MNS}$ \\
\hline$K A L$ & Kallikrein & M19647 & $\begin{array}{l}\text { AAATGCGAAAGTGTCTTGGC } \\
\text { ACAAAGTTATGGGATGGCAG }\end{array}$ & $\mathrm{WKY}<\mathrm{BN}=\mathrm{MNS}<\mathrm{R}=\mathrm{SHR}<\mathrm{S}=\mathrm{LEW}=\mathrm{AS}$ \\
\hline$L S N$ & Leukosianin & & Research Genetics & $\mathrm{S}=\mathrm{R}=\mathrm{LEW}=\mathrm{BN}=\mathrm{MNS}=\mathrm{AS}<\mathrm{WKY}=\mathrm{SHR}$ \\
\hline$M T 1 P A$ & $\begin{array}{r}\text { Metallothionein, } \\
\text { pseudogene A }\end{array}$ & & Research Genetics & $\mathrm{BN}=\mathrm{LEW}=\mathrm{WKY}=\mathrm{AS}<\mathrm{S}=\mathrm{R}=\mathrm{SHR}=\mathrm{MNS}$ \\
\hline$M Y L 2$ & $\begin{array}{l}\text { Myosin light } \\
\text { chain, muscle }\end{array}$ & X00975 & $\begin{array}{l}\text { TATAACCCCAGAAGAACTGCCC } \\
\text { ACCTGTAGTTGGAATGAGAAGC }\end{array}$ & $\mathrm{S}=\mathrm{R}=\mathrm{MNS}<\mathrm{WKY}=\mathrm{SHR}<\mathrm{BN}=\mathrm{LEW}=\mathrm{AS}$ \\
\hline$P B P C 2 *$ & $\begin{array}{l}\text { Prostatic binding } \\
\text { protein, } \mathrm{C} 2\end{array}$ & X05034 & $\begin{array}{l}\text { TGTGTCAGACAAGAAGTTCG } \\
\text { CACACTTGGCAAATTCCTTTCC }\end{array}$ & $\mathrm{S}=\mathrm{R}=\mathrm{BN}=\mathrm{LEW}=\mathrm{WKY}=\mathrm{SHR}=\mathrm{AS}<\mathrm{MNS}$ \\
\hline$P K C$ & $\begin{array}{l}\text { Protein kinase } \mathrm{C} \\
\text { type I }\end{array}$ & M13707 & $\begin{array}{l}\text { AGAACCCTTCACTGCTCACC } \\
\text { TGAGAAAGTCCCAGAAAGTGGC }\end{array}$ & $\mathrm{S}=\mathrm{R}=\mathrm{WKY}=\mathrm{MNS}<\mathrm{AS}<\mathrm{BN}=\mathrm{LEW}=\mathrm{SHR}$ \\
\hline$P T H^{*}$ & $\begin{array}{l}\text { Parathyroid } \\
\text { hormone }\end{array}$ & K01266 & $\begin{array}{l}\text { TTCCTCTGTGTGCATGAG } \\
\text { CAAACAGCAAGCCTTAGG }\end{array}$ & $\mathrm{BN}=\mathrm{MNS}<\mathrm{S}=\mathrm{R}=\mathrm{LEW}=\mathrm{WKY}=\mathrm{SHR}=\mathrm{AS}$ \\
\hline $\mathrm{R} 179 * \S$ & $\begin{array}{l}\text { Random cloned } \\
\text { SSR }\end{array}$ & & $\begin{array}{l}\text { CGTTCACCGCTTTGTGTC } \\
\text { CTCTGTGCTTGCTACTGTCC }\end{array}$ & $\mathrm{BN}<\mathrm{S}=\mathrm{LEW}=\mathrm{WKY}<\mathrm{R}<\mathrm{SHR}<\mathrm{AS}<\mathrm{MNS}$ \\
\hline $\mathrm{R} 191^{\S}$ & $\begin{array}{l}\text { Random cloned } \\
\text { SSR }\end{array}$ & & $\begin{array}{l}\text { TTCGGACTCAGTCTTGATTTGG } \\
\text { GCCTTCCTAGAATACTTGGT }\end{array}$ & $\mathrm{BN}=\mathrm{AS}<\mathrm{S}=\mathrm{R}=\mathrm{MNS}<\mathrm{LEW}<\mathrm{WKY}=\mathrm{SHR}$ \\
\hline $\mathrm{R} 197^{\S}$ & $\begin{array}{l}\text { Random cloned } \\
\text { SSR }\end{array}$ & & $\begin{array}{l}\text { ATTGTATATTCCAGACTAGC } \\
\text { AATTGATGTGACATTATTTTCAT }\end{array}$ & $\mathrm{S}=\mathrm{MNS}=\mathrm{AS}<\mathrm{R}=\mathrm{BN}<\mathrm{LEW}=\mathrm{WKY}=\mathrm{SHR}$ \\
\hline $\mathrm{R} 401^{\S}$ & $\begin{array}{l}\text { Random cloned } \\
\text { SSR }\end{array}$ & & $\begin{array}{l}\text { TGAGCCTGGGCACTATGTAG } \\
\text { GGACAGGGACTGGAATCATC }\end{array}$ & $\mathrm{LEW}=\mathrm{BN}=\mathrm{AS}<\mathrm{S}=\mathrm{R}=\mathrm{MNS}<\mathrm{WKY}=\mathrm{SHR}$ \\
\hline $\mathrm{R} 416^{\S}$ & $\begin{array}{l}\text { Random cloned } \\
\text { SSR }\end{array}$ & & $\begin{array}{l}\text { GGTATTGCATGGCTATGAGG } \\
\text { GCAGCCATAGAGTGCCAAC }\end{array}$ & $\mathrm{LEW}=\mathrm{BN}=\mathrm{WKY}<\mathrm{SHR}<\mathrm{S}=\mathrm{R}=\mathrm{MNS}=\mathrm{AS}$ \\
\hline RCA01.20 & $\begin{array}{l}\text { Random cloned } \\
\text { SSR }\end{array}$ & & $\begin{array}{l}\text { GTAGGTGTAGAAAAGATGCTGC } \\
\text { ATCAATGGAGGCTCTGATGGG }\end{array}$ & $\mathrm{LEW}=\mathrm{BN}=\mathrm{WKY}=\mathrm{MNS}<\mathrm{S}=\mathrm{R}=\mathrm{AS}<\mathrm{SHR}$ \\
\hline RCA07.06 & $\begin{array}{l}\text { Random cloned } \\
\text { SSR }\end{array}$ & & $\begin{array}{l}\text { CСААТTTCTCAACATCACCCC } \\
\text { CAATATCCTATGATAGATGATGG }\end{array}$ & $\mathrm{BN}=\mathrm{AS}<\mathrm{R}<\mathrm{S}=\mathrm{WKY}<\mathrm{LEW}=\mathrm{SHR}=\mathrm{MNS}$ \\
\hline RCA09.01 & $\begin{array}{l}\text { Random cloned } \\
\text { SSR }\end{array}$ & & $\begin{array}{l}\text { СССCATCTATCTATCCAACGG } \\
\text { CTCTGGGATGCTTTGTGAAGG }\end{array}$ & $\mathrm{MNS}<\mathrm{AS}<\mathrm{S}=\mathrm{R}=\mathrm{WKY}=\mathrm{SHR}<\mathrm{LEW}<\mathrm{BN}$ \\
\hline RCA17.42 & $\begin{array}{l}\text { Random cloned } \\
\text { SSR }\end{array}$ & & $\begin{array}{l}\text { GTAGTTGTGACTGCCTTTCCTG } \\
\text { GATGAGAACATCCTGGAGAATG }\end{array}$ & $\mathrm{S}=\mathrm{R}=\mathrm{WKY}=\mathrm{SHR}=\mathrm{MNS}=\mathrm{AS}<\mathrm{LEW}=\mathrm{BN}$ \\
\hline RCA24.16 ${ }^{\S}$ & $\begin{array}{l}\text { Random cloned } \\
\text { SSR }\end{array}$ & & $\begin{array}{l}\text { ATCACTTCTGACCAGAGGACC } \\
\text { TCACTTTCACATGACAGACCC }\end{array}$ & $\mathrm{WKY}=\mathrm{SHR}<\mathrm{MNS}<\mathrm{S}=\mathrm{R}=\mathrm{LEW}=\mathrm{BN}=\mathrm{AS}$ \\
\hline$S A(\mathrm{PSA} 1)$ & $\begin{array}{l}\text { Anonymous } \\
\text { SA gene }\end{array}$ & U04637 & $\begin{array}{l}\text { CCTACACAGCAAGTTCCAGAGC } \\
\text { TACСCCСATTGCCTATCTCC }\end{array}$ & $\mathrm{S}=\mathrm{SHR}=\mathrm{MNS}=\mathrm{AS}<\mathrm{R}<\mathrm{BN}<\mathrm{LEW}=\mathrm{WKY}$ \\
\hline$S A(\mathrm{PSA} 2)$ & $\begin{array}{l}\text { Anonymous } \\
\text { SA gene }\end{array}$ & U04638 & $\begin{array}{l}\text { CCTAAATTAGCTTGTAGGGAGG } \\
\text { TACCTAGCCTTAGGGATTTGC }\end{array}$ & $\mathrm{S}=\mathrm{R}=\mathrm{SHR}=\mathrm{MNS}=\mathrm{AS}<\mathrm{BN}<\mathrm{LEW}=\mathrm{WKY}$ \\
\hline
\end{tabular}




\begin{tabular}{|c|c|c|c|c|}
\hline Locus name & Gene name & $\begin{array}{c}\text { GenBank } \\
\text { accession number }\end{array}$ & Primers $\left(5^{\prime}-3^{\prime}\right)$ & $\begin{array}{l}\text { Length variation of the PCR-product } \\
\text { among } 8 \text { inbred strains }\end{array}$ \\
\hline$S A(\mathrm{PSA} 3)$ & $\begin{array}{l}\text { Anonymous } \\
\text { SA gene }\end{array}$ & U04639 & $\begin{array}{l}\text { TGTTACACTCAGGCTTCTATCC } \\
\text { АTTCTCTTCCGAAAAAGC }\end{array}$ & $\mathrm{LEW}=\mathrm{WKY}<\mathrm{S}=\mathrm{R}=\mathrm{SHR}=\mathrm{MNS}=\mathrm{AS}<\mathrm{BN}$ \\
\hline
\end{tabular}

SSR, simple sequence repeat. STMS, sequence tagged microsatellite site. Primers were designed using the Primer Detective Program (Clontech, Palo Alto, CA) except CYPE (20), CYP2A3 (22), and D1N64 (23). D1Mco markers were developed by screening the flow-sorted chromosome 1 library with (CA) 15 and $(\mathrm{CT})_{15}$ oligonucleotides. Primers purchased from Research Genetics (Huntsville, Alabama) were D7Mits which are mouse MapPair primers and D1Mits and D1Mghs which are rat MapPair primers. *D0Mco1, D0Mco3, D1Mgh1, D1Mgh15, D1Mgh17, D1Mit2, D1Mit10, D1Mit15, $P B P C 2, P T H$ and R179 could not be placed on the map in Fig. 1 because these markers were not polymorphic among S, LEW, and WKY rats. ${ }^{*}$ These markers were either reported to be, or were thought to be, on rat chromosome 1, but they were not linked to any marker of the linkage group shown in Fig. 1; they are D0Mco2, D0Mco4, D1Mgh18, D7Mit62, D7Mit89, and EN3C. ${ }^{\S}$ Primers developed at the University of Oxford, England.

$\mathrm{F}_{2}$ populations were weaned at $30 \mathrm{~d}$ of age and placed on an $8 \% \mathrm{NaCl}$ diet (Teklad diet 82050; Teklad Premier Laboratory Diets, Madison, WI) at $37 \mathrm{~d}$ of age. When the highest blood pressures had reached $>200 \mathrm{mmHg}$, the blood pressures of the rats were measured several times during a 7-10-d period. Systolic blood pressure was taken by the tail cuff method on the conscious restrained rat (15) (IITC, Inc., Woodland Hills, CA) with the ambient temperature maintained at $29^{\circ} \mathrm{C}$. At least three consecutive consistent readings were taken at a given session and averaged as that session's reading. Blood pressures at three separate sessions separated by $2 \mathrm{~d}$ were taken. These three separate session's pressures were averaged as the final blood pressure.

The rats were killed with an overdose of sodium pentobarbital. Pieces of liver were stored at $-70^{\circ} \mathrm{C}$. DNA for genotyping was extracted from frozen livers by the method of Blin and Stafford (16).

Markers from chromosome 1-sorted DNA. Rat chromosome 1 DNA was obtained by flow sorting as described by Hoebee et al. (17) and amplified by an initial PCR using a degenerate oligonucleotideprimer (DOP) (6-MW primer: 5'-CCGACTCGAGNNNNNNATGTGG-3') as described by Telenius et al. $(18,19)$. Reamplification was carried out in a total vol of $100 \mu$ l reaction mixture containing $5 \mu \mathrm{l}$ PCR products from the previous round of amplification, $1 \times$ PCR buffer, $200 \mu \mathrm{M}$ of each of the four dNTPs, $800 \mathrm{nM}$ of $6-\mathrm{MW}$ primer, and 1.25 U of Taq DNA polymerase (Perkin-Elmer Cetus, Norwalk, CT). After an initial 5-min denaturation at $95^{\circ} \mathrm{C}$ the reaction was subjected to 35 cycles of $1 \mathrm{~min}$ at $94^{\circ} \mathrm{C}, 2 \mathrm{~min}$ at $55^{\circ} \mathrm{C}, 3 \mathrm{~min}$ at $72^{\circ} \mathrm{C}$, followed by an additional $10 \mathrm{~min}$ at $72^{\circ} \mathrm{C}$ for extension. $20 \mu \mathrm{l}$ of PCR products were subjected to an $0.8 \%$ agarose gel electrophoresis to check if the DOP-PCR worked. If the DOP-PCR works, a smear of DNA between 250 bp to 1,500 bp of DNA is observed.

$75 \mu \mathrm{l}$ of DOP-PCR-amplified rat chromosome-1 DNA was digested with XhoI restriction enzyme in a total volume of $100 \mu \mathrm{l}$ at $37^{\circ} \mathrm{C}$ for $3 \mathrm{~h}$ (there is an Xhol site in the $5^{\prime}$-end of the $6-\mathrm{MW}$ primer). The digested DNA was concentrated in a centricon-30 microconcentrator (Amicon, Inc., Beverly, MA) by centrifugation at 1,000 $\mathrm{g}$ for $2 \mathrm{~h}$ to get about $45 \mu \mathrm{l}$ of concentrated DNA. This DNA was cloned into the SalI site of the vector pT7T3 U18 plasmid (SalI and XhoI yield compatible ends). Clones were screened with ${ }^{32} \mathrm{P}$ end-labeled $(\mathrm{CA})_{15}$ and $(\mathrm{CT})_{15}$ oligonucleotides. The positive clones were purified by two additional rounds of screening and sequenced. PCR primers were designed using the Primer Detective Program (Clontech, Palo Alto, CA). Markers developed by this method are given in Table I and were: D1Mco1, D1Mco2, D1Mco3, D1Mco4, D1Mco5, D1Mco6, D0Mco1, D0Mco2, D0Mco3, D0Mco4.

Additional markers. New microsatellite based markers were also obtained from a small insert library by screening with $(\mathrm{CA})_{15}$ and $(\mathrm{CT})_{15}$ oligonucleotides. Markers that were linked to known markers on chromosome 1 in various segregating rat populations were used here. These markers are given in Table I and were: R179, R191, R197, R401, R416, RCA01.20, RCA07.06, RCA09.01, RCA17.42, RCA24.16.

Markers were also designed around microsatellite sequences in
GenBank based on information from reference [20]; see Table I for locus designation and GenBank accession number. Rat Map Pair primers (21) (Research Genetics, Huntsville, AL) are listed in Table I. Mouse chromosome 7 has homology with rat chromosome 1; and nine mouse chromosome 7 markers (D7Mit45, D7Mit62, D7Mit66, D7Mit69, D7Mit87, D7Mit89, D7Mit99, D7Mit101, D7Mit109) (Research Genetics) were found to be useful in the rat. Primers for $C Y P E, C Y P 2 A 3$, and D1N64 were from references $(20,22,23)$, respectively. Microsatellites for the $S A$ gene were recently published (24). Rats used here had also been previously typed at the GLUTB and HITH loci (25) on rat chromosomes 5 and 17, respectively.

Genotyping. Genotyping was done by PCR amplification of DNA around microsatellites. PCR reaction vol was $25 \mu \mathrm{l}$ which contained $1 \times$ PCR buffer, $1-2.5 \mathrm{mM} \mathrm{MgCl}_{2}, 200 \mu \mathrm{M}$ of each the four dNTPs, $1 \mu \mathrm{M}$ of each primer, $0.625 \mathrm{U}$ Taq polymerase (Perkin-Elmer Cetus), and 20-200 ng rat genomic DNA. PCR was started at $95^{\circ} \mathrm{C}$ for $5 \mathrm{~min}$ and continued for $25-35$ cycles of $94^{\circ} \mathrm{C}$ for $1 \mathrm{~min}, 45-65^{\circ} \mathrm{C}$ (depending on the primer) for $2 \mathrm{~min}, 72^{\circ} \mathrm{C}$ for $2 \mathrm{~min}$, followed by additional $72^{\circ} \mathrm{C}$ for $10 \mathrm{~min}$. The PCR products were subjected to electrophoresis on a $4 \%$ agarose gel which contained $1 \mathrm{mg} / \mathrm{ml}$ ethidium bromide for photographing the PCR products under the ultraviolet light. In cases where the PCR allelic products were too close to separate on an agarose gel, a polyacrylamide sequencing gel was used. In this case one of the primers was end-labeled with $\gamma^{32} \mathrm{P}$-ATP with T4 polynucleotide kinase and used in the PCR reaction without purification. After PCR the reaction was denatured by adding an equal volume of formamide containing $0.1 \%$ bromphenol blue and $0.1 \%$ xylene cyanol and heating at $100^{\circ} \mathrm{C}$ for $2 \mathrm{~min}$, cooling on ice, and $4 \mu \mathrm{l}$ was loaded onto an $8 \%$ polyacrylamide sequencing gel. After electrophoresis the gel was dried and exposed $2-14 \mathrm{~h}$ at $-80^{\circ} \mathrm{C}$ to X-OMAT AR film (Eastman Kodak, Rochester, NY) using a fluorescent screen, and developed.

Linkage and statistical analysis. Cosegregation of blood pressure (body weight or heart weight) with alleles at a marker locus was evaluated by comparing blood pressures among genotypes at each locus by a one-way ANOVA using SPSS programs (SPSS, Chicago, IL). When testing for dominance of an allele at a given locus the one-way ANOVA was followed by a contrast that compared the average value of blood pressure of the two homozygous groups to that of the heterozygotes. Linkage maps and QTL localization were done with the MAPMAKER Programs $(26,27)$ obtained from Dr. Eric Lander (Whitehead Institute, Cambridge, MA). The MAPMAKER programs also detect potential genotyping errors based on results of flanking markers. These samples were always retyped to confirm or correct the results.

\section{Results}

Fig. 1 shows the genetic maps constructed for the $\mathrm{F}_{2}(\mathrm{WKY} \times$ $\mathrm{S})$ and $\mathrm{F}_{2}(\mathrm{LEW} \times \mathrm{S})$ populations and the composite map for 


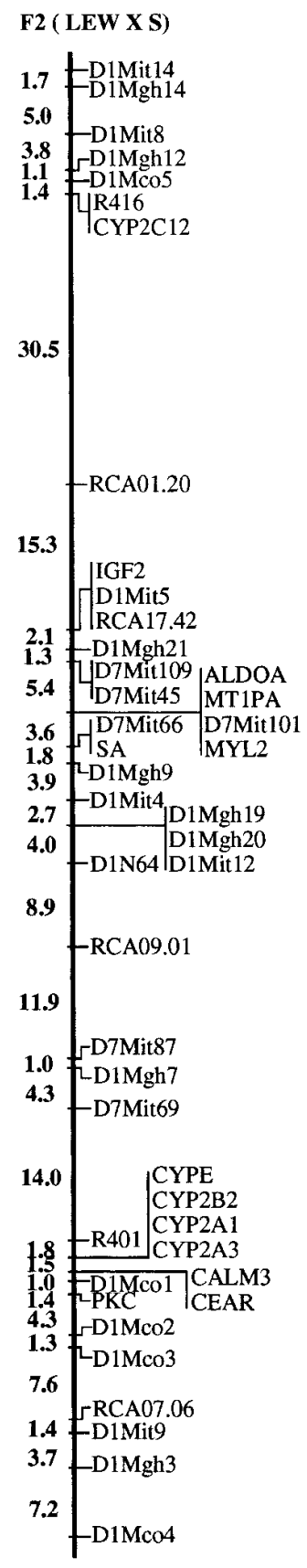

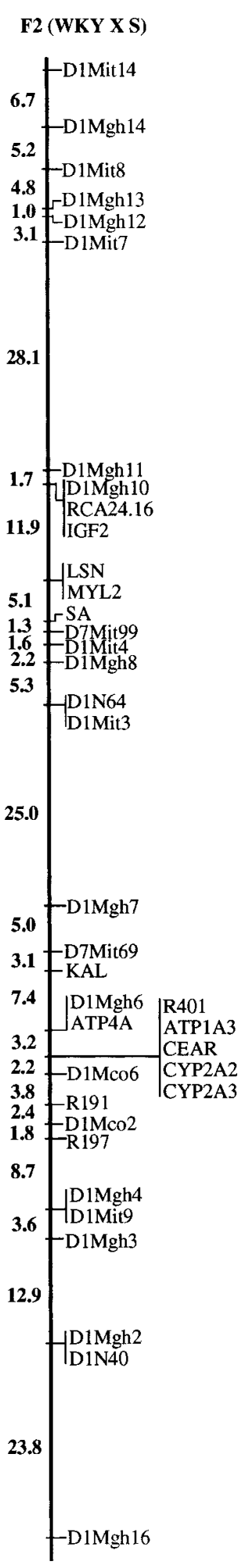

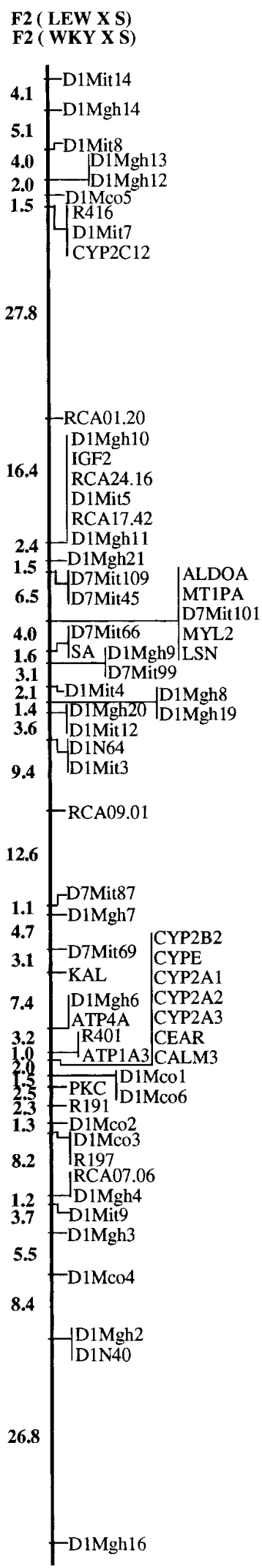

Figure 1. Linkage maps for rat chromosome 1 . The left panel is the map for an $\mathrm{F}_{2}(\mathrm{LEW} \times \mathrm{S})$ population of 151 rats, center panel for an $\mathrm{F}_{2}(\mathrm{WKY} \times \mathrm{S})$ population of 159 rats, and the right panel is a map for the two populations combined. The position of the centromere is unknown. Genetic distances are in centiMorgans using the Haldane correction. The map was constructed using the MAPMAKER programs $(26,27)$. the combined populations. Table II gives the blood pressure of each of the three genotypes segregating at selected loci on chromosome 1 for the $\mathrm{F}_{2}(\mathrm{LEW} \times \mathrm{S})$ population. The analysis of this blood pressure data for the $\mathrm{F}_{2}(\mathrm{LEW} \times \mathrm{S})$ population is summarized in Fig. 2 in conjunction with a map of selected loci for chromosome 1 .

Note in Fig. 2 that the blood pressure effect (the blood pressure of rats homozygous [SS] for the S-rat allele minus the 


\begin{tabular}{l|llll}
$\begin{array}{l}\text { Rat Chromosome 1 } \\
\text { Linkage Map }\end{array}$ & $\begin{array}{l}\text { Blood } \\
\text { Pressure } \\
\text { Effect }\end{array}$ & \multicolumn{2}{l}{ One-way ANOVA } & QTL \\
Location
\end{tabular}

Figure 2. Linkage map of rat chromosome 1 and associated blood pressure linkage data for the $\mathrm{F}_{2}(\mathrm{LEW} \times \mathrm{S})$ population of 151 rats using selected markers from Fig. 1 . Rats were raised on $8 \% \mathrm{NaCl}$ diet for $9 \mathrm{wk}$ starting at $37 \mathrm{~d}$ of age. Genetic distances are $\mathrm{cM}$ with the Haldane correction; the position of the centromere is unknown. "Blood pressure effect" is the difference in blood pressure between rats homozygous for the S-rat allele, and those homozygous for the Lewis-rat allele at a given marker. Values for the $\mathrm{F}$ statistic $(F)$ and probability $(P)$ from an ANOVA are given to evaluate the effect of genotype at each locus on blood pressure. "QTL location" and the map itself were generated using the MAPMAKER/QTL programs $(26,27)$. The arrow indicates the position of the maximum LOD score and the vertical bar indicates \pm 1 LOD for linkage to blood pressure.

blood pressure of rats homozygous [ $L L]$ for the Lewis-rat allele) is positive and reaches a maximum in the region of the $S A$ gene. The values decrease in the middle of the map and then increase markedly toward the bottom of the map. The F statistic and the associated probability value from a one-way ANOVA at each locus mirror these changes, the most significant effects occurring near the $S A$ locus $(P=0.0045)$ and near the D1Mco1 locus $(P=0.0006)$. The $S A$ and D1Mco1 loci are $\sim 55 \mathrm{cM}$ apart (using the Haldane mapping function), which is far enough to segregate essentially independently of each
Table II. Systolic Blood Pressure by Genotype for Selected Loci on Rat Chromosome 1 in the $F_{2}($ Lew $\times S)$ Population Raised on $8 \% \mathrm{NaCl}$ Diet

\begin{tabular}{|c|c|c|c|}
\hline \multirow[b]{2}{*}{ Locus } & \multicolumn{3}{|c|}{ Blood Pressure by Genotype } \\
\hline & SS & $S L$ & $L L$ \\
\hline D1Mit14 & $190.6 \pm 6.32(38)$ & $185.7 \pm 3.93(71)$ & $190.7 \pm 5.66(40)$ \\
\hline D1Mit8 & $190.3 \pm 6.03(39)$ & $185.3 \pm 4.08(69)$ & $191.1 \pm 5.29(43)$ \\
\hline$C Y P 2 C 12$ & $193.5 \pm 6.11(37)$ & $186.2 \pm 4.02(74)$ & $187.1 \pm 5.40(40)$ \\
\hline RCA01.20 & $193.0 \pm 5.99(34)$ & $189.3 \pm 4.07(77)$ & $182.2 \pm 5.33(40)$ \\
\hline$I G F 2$ & $191.8 \pm 5.17(47)$ & $189.8 \pm 4.40(69)$ & $180.5 \pm 5.22(35)$ \\
\hline$M Y L 2$ & $200.7 \pm 5.30(48)$ & $185.5 \pm 4.33(72)$ & $175.5 \pm 3.63(31)$ \\
\hline$S A$ & $200.7 \pm 5.18(50)$ & $184.7 \pm 4.55(68)$ & $176.7 \pm 3.20(33)$ \\
\hline D1Mit4 & $197.8 \pm 5.07(55)$ & $185.6 \pm 4.52(66)$ & $176.6 \pm 3.44(30)$ \\
\hline D1N64 & $197.8 \pm 5.38(49)$ & $183.2 \pm 4.20(69)$ & $184.6 \pm 5.02(33)$ \\
\hline RCA09.01 & $198.1 \pm 5.31(53)$ & $187.7 \pm 4.24(64)$ & $175.5 \pm 4.17(33)$ \\
\hline D7Mit87 & $195.0 \pm 5.76(44)$ & $189.5 \pm 3.83(80)$ & $175.7 \pm 5.24(26)$ \\
\hline D7Mit69 & $196.9 \pm 5.90(42)$ & $190.2 \pm 3.89(79)$ & $170.9 \pm 4.55(30)$ \\
\hline R401 & $202.6 \pm 6.13(38)$ & $187.0 \pm 3.69(78)$ & $175.5 \pm 5.48(35)$ \\
\hline D1Mco1 & $205.6 \pm 6.34(38)$ & $185.7 \pm 3.51(78)$ & $175.2 \pm 5.62(34)$ \\
\hline D1Mco2 & $203.8 \pm 6.88(36)$ & $186.9 \pm 3.46(74)$ & $179.7 \pm 5.61(37)$ \\
\hline RCA07.06 & $196.2 \pm 6.23(41)$ & $187.6 \pm 3.75(73)$ & $181.5 \pm 4.90(35)$ \\
\hline D1Mgh3 & $192.8 \pm 6.69(35)$ & $190.5 \pm 3.90(75)$ & $178.1 \pm 4.73(40)$ \\
\hline D1Mco4 & $195.2 \pm 6.85(33)$ & $188.5 \pm 4.02(73)$ & $182.8 \pm 4.85(45)$ \\
\hline
\end{tabular}

Blood pressure data is mean $\pm \mathrm{SE}$ in $\mathrm{mmHg}$ for each genotype. The number of rats in each genotypic class is given in parenthesis. $S$, allele for S rats, $L$, allele for LEW rats. See Table I for locus designations, and Fig. 2 for statistical analysis of blood pressure data.

other. The MAPMAKER/QTL program gave LOD score peaks for linkage to blood pressure in the region of the $S A$ and D1Mco1 loci of 2.45 and 3.38, respectively.

There was a strong correlation in the $\mathrm{F}_{2}(\mathrm{LEW} \times \mathrm{S})$ population between heart weight and blood pressure (Fig. 3). A linkage analysis of heart wt, body wt and heart wt/body wt ratio with selected loci along chromosome 1 is given in Table III. Heart weight showed basically the same pattern as blood pressure. The $S$ alleles increased heart weight in two regions, one around the $S A$ locus and a second region around the D1Mco1 locus. Note, however, that the effect on heart wt of the $S$ allele at the $S A$ locus $(+82.3 \mathrm{mg}, P=0.039)$ appears relatively weak compared to the effect of the D1Mco1 locus $(+130.4 \mathrm{mg}, P=$ 0.0036). This could be due to the lower blood pressure associated with the SA region compared to D1Mco1. It could also be related to the fact that the $S$ allelic region around the $S A$ locus had a negative effect on body wt, which would be expected to reduce absolute heart size. Correcting heart wt for differences in body wt by using the heart wt/body wt ratio in Table III yielded a very strong and positive effect of the $\mathrm{S}$ allelic region around the $S A$ locus on heart wt/body wt ratio. Using body wt as a covariate in an ANOVA yielded a more significant $(P=$ 0.001 ) effect of the $S A$ region on heart wt than did the analysis of uncorrected heart wt.

It was possible to test both the chromosome 1 region marked by the $S A$ locus and the new chromosome 1 blood pressure QTL region marked by D1Mco1 for linkage to blood pressure in populations derived from crosses of $\mathrm{S}$ rats with various normotensive strains. The $S A$ locus cosegregated with 


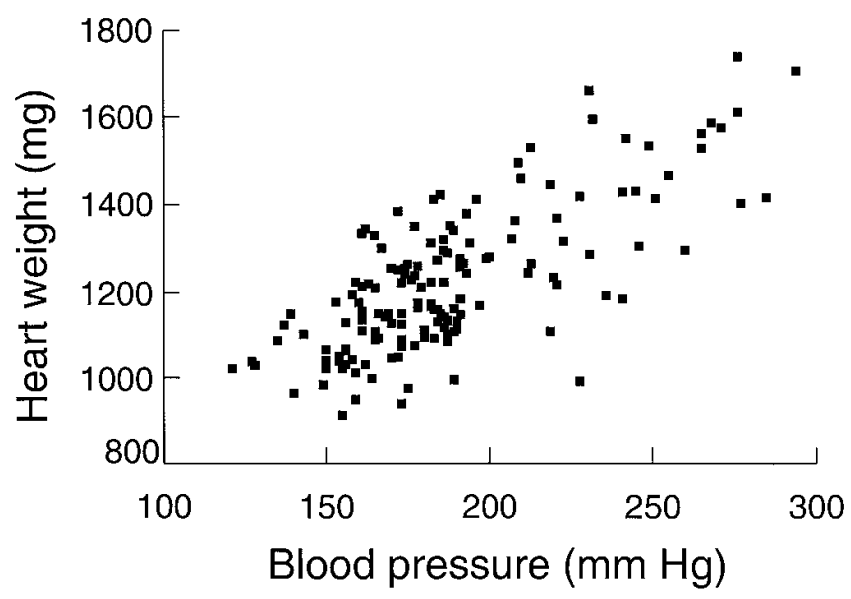

Figure 3. Relationship between heart weight and blood pressure in the $\mathrm{F}_{2}(\mathrm{LEW} \times \mathrm{S})$ population raised on $8 \% \mathrm{NaCl}$ diet for $9 \mathrm{wk}$ starting at $37 \mathrm{~d}$ of age. $r=0.75, P<0.001, n=151$ rats.

blood pressure only in the $\mathrm{F}_{2}(\mathrm{LEW} \times \mathrm{S})$ population, but not in $\mathrm{F}_{2}(\mathrm{WKY} \times \mathrm{S})$ or in $\mathrm{F}_{2}(\mathrm{R} \times \mathrm{S})$ or in a backcross to the $\mathrm{S}$, $\mathrm{F}_{1}(\mathrm{R} \times \mathrm{S}) \times \mathrm{S}$ (Table IV). Using loci near D1Mco1 $(C Y P 2 A 3$ and $C Y P 2 B 2$ were used), five $\mathrm{F}_{2}$ populations were tested for linkage of this chromosomal region to blood pressure. Only the $\mathrm{F}_{2}(\mathrm{LEW} \times \mathrm{S})$ gave a clearly significant linkage to blood pressure (Table $\mathrm{V}$ ).

The $\mathrm{F}_{2}(\mathrm{LEW} \times \mathrm{S})$ population is remarkable because, besides the two QTL on chromosome 1, we know of two other chromosome regions with substantial effects on blood pressure. These are marked by GLUTB on chromosome 5, and HITH on chromosome 17, as reported previously (25). The effects of all four chromosomal regions (marked by $S A$, D1Mco1, GLUTB, and HITH) on blood pressure and heart weight are summarized in Tables VI and VII, respectively. There were no interactions between any two of these loci on either blood pressure or heart weight by two-way factorial ANOVA analyzing the loci pairwise, or in a four-way factorial analysis with all four loci simultaneously. The effects of these loci are therefore additive, or, at least they are additive on the genetic background of the $\mathrm{F}_{2}(\mathrm{LEW} \times \mathrm{S})$ population. As shown in Tables VI and VII these four loci accounted for $106 \mathrm{mmHg}$ and $38 \%$ of total variance for blood pressure, and $506 \mathrm{mg}$ of heart wt (the average heart wt for all rats in the population was $1,217 \mathrm{mg}$ ) and $34 \%$ of total variance for heart wt.

The frequency distribution for blood pressure in the $\mathrm{F}_{2}(\mathrm{LEW} \times \mathrm{S})$ population was significantly skewed to the right; the $\mathrm{g}_{1}$ statistic for skewing was 0.98 which is statistically significant $(P<0.005)$. Correcting for the blood pressure effects of genotypes at the $S A$, D1Mco1, GLUTB, and HITH loci yielded a "corrected" distribution which was still skewed to the right $\left(\mathrm{g}_{1}=0.41,0.025<P<0.05\right)$ but not as badly as the uncorrected distribution. The variance of the uncorrected blood pressure distribution was $1,226(\mathrm{mmHg})^{2}$ and for the corrected distribution $883(\mathrm{mmHg})^{2}$. Because the uncorrected blood pressure distribution was skewed to the right, all ANOVAs reported above were repeated on log transformed data and by a nonparametric Kruskal-Wallis one-way ANOVA. Neither procedure altered the conclusions given above.

Table III. Analysis of the Effect of Rat Chromosome 1 on Body Weight (BW), Heart Weight (HW), and Heart Weight/Body Weight Ratio for the Male $F_{2}(L E W \times S)$ Population Raised on $8 \% \mathrm{NaCl}$ Diet

\begin{tabular}{|c|c|c|c|c|c|c|c|c|c|}
\hline \multirow[b]{2}{*}{ Locus } & \multicolumn{3}{|c|}{ HW } & \multicolumn{3}{|c|}{ BW } & \multicolumn{3}{|c|}{ HW/BW Ratio } \\
\hline & Effect & $\mathrm{F}$ & $\mathrm{P}$ & Effect & $\mathrm{F}$ & $\mathrm{P}$ & Effect & $\mathrm{F}$ & $\mathrm{P}$ \\
\hline & $m g$ & & & $g$ & & & $m g / g$ & & \\
\hline D1Mit14 & +33.4 & 1.44 & 0.24 & -7.8 & 1.12 & 0.33 & +0.176 & 1.56 & 0.21 \\
\hline D1Mit8 & +41.9 & 1.41 & 0.25 & -6.8 & 0.72 & 0.49 & +0.183 & 2.45 & 0.090 \\
\hline$C Y P 2 C 12$ & +64 & 2.50 & 0.086 & -13.4 & 2.34 & 0.095 & +0.336 & 5.91 & 0.0034 \\
\hline RCA01.20 & +57.7 & 1.08 & 0.34 & -13.3 & 2.68 & 0.072 & +0.291 & 3.22 & 0.043 \\
\hline$I G F 2$ & +36.8 & 0.48 & 0.62 & -17.2 & 4.44 & 0.013 & +0.274 & 3.21 & 0.043 \\
\hline$M Y L 2$ & +71.4 & 2.66 & 0.073 & -18.7 & 4.95 & 0.0083 & +0.408 & 8.23 & 0.0004 \\
\hline$S A$ & +82.3 & 3.36 & 0.037 & -18.0 & 4.80 & 0.0096 & +0.427 & 9.38 & 0.0001 \\
\hline D1Mit4 & +82.7 & 3.78 & 0.025 & -14.3 & 2.76 & 0.066 & +0.392 & 8.40 & 0.0004 \\
\hline D1N64 & +38.5 & 2.48 & 0.087 & -17.6 & 4.78 & 0.0097 & +0.326 & 7.62 & 0.0007 \\
\hline RCA09.01 & +64.9 & 1.93 & 0.15 & -16.9 & 5.50 & 0.0050 & +0.377 & 8.35 & 0.0004 \\
\hline D7Mit87 & +50.6 & 0.90 & 0.41 & -12.1 & 3.11 & 0.047 & +0.296 & 2.84 & 0.062 \\
\hline D7Mit69 & +78.3 & 3.25 & 0.041 & -6.9 & 2.43 & 0.092 & +0.329 & 3.98 & 0.021 \\
\hline $\mathrm{R} 401$ & +106.6 & 3.76 & 0.025 & -5.0 & 0.38 & 0.68 & +0.365 & 5.05 & 0.0076 \\
\hline D1Mco1 & +130.4 & 5.84 & 0.0036 & -4.1 & 0.23 & 0.79 & +0.418 & 6.72 & 0.0016 \\
\hline D1Mco2 & +111.8 & 4.26 & 0.016 & -4.9 & 0.42 & 0.66 & +0.368 & 5.38 & 0.0056 \\
\hline RCA07.06 & +65.2 & 1.44 & 0.24 & -10.5 & 2.33 & 0.10 & +0.291 & 3.39 & 0.036 \\
\hline D1Mgh3 & +76.0 & 3.13 & 0.047 & -11.1 & 2.02 & 0.14 & +0.340 & 5.36 & 0.0057 \\
\hline D1Mco4 & +51.1 & 1.03 & 0.36 & -9.7 & 1.25 & 0.29 & +0.247 & 2.42 & 0.092 \\
\hline
\end{tabular}

"Effect" is the difference in the phenotypic trait mean of homozygous $S S$ rats minus the phenotypic trait mean of homozygous $L L$ rats at each locus where $S$ is the allele from $\mathrm{S}$ rats and $L$ is the allele from Lewis rats. F is the F statistic, and P the probability, from a one-way analysis of variance of phenotype by genotype at each locus. 
Table IV. Cosegregation of Systolic Blood Pressure with Genotypes at the SA Locus in Various Segregating Populations Raised on $8 \% \mathrm{NaCl}$

\begin{tabular}{|c|c|c|c|c|c|}
\hline \multirow[t]{2}{*}{ Population } & \multicolumn{3}{|c|}{ Blood pressure by $S A$ genotype } & \multirow[t]{2}{*}{ Blood pressure effect } & \multirow[t]{2}{*}{$\begin{array}{c}\text { One-way ANOVA } \\
\text { probability }\end{array}$} \\
\hline & SS & $S L$ & $L L$ & & \\
\hline $\mathrm{F}_{2}(\mathrm{LEW} \times \mathrm{S}) *$ & $200.7 \pm 5.18(50)$ & $184.7 \pm 4.55(68)$ & $176.7 \pm 3.20(33)$ & +24.0 & 0.0045 \\
\hline $\mathrm{F}_{2}(\mathrm{WKY} \times \mathrm{S})^{\ddagger}$ & $\begin{array}{c}S S \\
177.0 \pm 4.32(40)\end{array}$ & $\begin{array}{c}S W \\
169.3 \pm 2.58(72)\end{array}$ & $\begin{array}{c}W W \\
179.9 \pm 3.89(44)\end{array}$ & -2.9 & 0.057 \\
\hline $\mathrm{F}_{2}(\mathrm{R} \times \mathrm{S})$ & $\begin{array}{c}S S \\
167.5 \pm 3.85(25)\end{array}$ & $\begin{array}{c}S R \\
161.8 \pm 2.67(56)\end{array}$ & $\begin{array}{c}R R \\
166.0 \pm 4.13(30)\end{array}$ & +1.5 & 0.44 \\
\hline $\mathrm{F}_{1}(\mathrm{R} \times \mathrm{S}) \times \mathrm{S}$ & $163.0 \pm 2.95(35)$ & $166.3 \pm 3.28(35)$ & & & 0.46 \\
\hline
\end{tabular}

Blood pressure data are mean \pm SE in mmHg for each genotype. $S, \mathrm{~S}$ allele; $L$, LEW allele; $W, \mathrm{WKY}$ allele; number of rats in each genotypic class are in parenthesis; "Blood Pressure Effect" is the difference in blood pressure of homozygous $S S$ minus blood pressure of homozygotes for the contrasting allele. *These data differ slightly from previous published data on this population (10) because the present data includes more rats; also genotyping errors based on restriction fragment length polymorphisms were corrected. ${ }^{*}$ These data were published previously (10), but are included here for comparative purposes.

\section{Discussion}

Identification of the chromosomal regions containing blood pressure QTL by linkage analysis is a fundamental first step in understanding genetic hypertension, but the statistical nature of this localization is limiting. The best that can be expected under reasonably favorable conditions (a QTL with a moderate to large effect, a dense genetic map, and a very large population) is to localize the QTL to a region $\sim 10 \mathrm{cM}$ in size (28). Thus, it is obvious that if two QTL are on the same chromosome and close together, problems arise in differentiating them.

Using the MAPMAKER/QTL program and simulated data, when two QTL are far apart they were readily recognizable (27). If the two QTL were closer, then simulated data predict that a "ghost" QTL between the actual QTL will be observed (29). Rat chromosome 1 in the $F_{2}(L E W \times S)$ population provides a reasonably favorable situation in that the two putative QTL are far enough apart to be recognized as distinct. The estimated distance between them is $55 \mathrm{cM}$ (Haldane corrected), which is presumably far enough apart for them to be differentiated statistically. Interference of the two putative QTL in our data, however, may account for the paradoxical increase in blood pressure effect at marker RCA09.01 in Fig. 2, or more likely it is just statistical variation. For comparative purposes the reader is referred to our previous work on rat chromosome 2 (30), which illustrates a reasonable genetic map showing only one QTL, and to work on rat chromosome 10 where there is evidence for two QTL too close to be adequately resolved (31).

In general it is desirable to have the linkage of a chromosomal region to the phenotypic trait supported by a LOD score of $\geq 3$ or a one-way ANOVA of $P \leq 0.001(2,27)$. This is because in searching for QTL so many markers are used that

Table V. Cosegregation of Systolic Blood Pressure with Genotypes at the CYP2A3 (or CYP2B2) Locus in Various $F_{2}$ Rat Populations Raised on $8 \% \mathrm{NaCl}$

\begin{tabular}{|c|c|c|c|c|c|}
\hline Population & \multicolumn{3}{|c|}{ Blood pressure by $C Y P 2 A 3$ genotype } & Blood pressure effect & $\begin{array}{c}\text { One-way ANOVA } \\
\text { probability }\end{array}$ \\
\hline $\mathrm{F}_{2}(\mathrm{LEW} \times \mathrm{S})$ & $\begin{array}{c}S S \\
203.7 \pm 6.00(41)\end{array}$ & $\begin{array}{c}S L \\
186.1 \pm 3.55(77)\end{array}$ & $\begin{array}{c}L L \\
174.2 \pm 5.72(33)\end{array}$ & +29.5 & 0.0009 \\
\hline $\mathrm{F}_{2}(\mathrm{WKY} \times \mathrm{S})$ & $\begin{array}{c}S S \\
178.1 \pm 5.95(30)\end{array}$ & $\begin{array}{c}S W \\
176.2 \pm 2.38(93)\end{array}$ & $\begin{array}{c}W W \\
164.7 \pm 3.20(36)\end{array}$ & +13.4 & 0.034 \\
\hline $\mathrm{F}_{2}(\mathrm{R} \times \mathrm{S})$ & $\begin{array}{c}S S \\
163.8 \pm 3.35(39)\end{array}$ & $\begin{array}{c}S R \\
163.4 \pm 3.06(47)\end{array}$ & $\begin{array}{c}R R \\
166.4 \pm 3.90(25)\end{array}$ & -2.6 & 0.83 \\
\hline $\mathrm{F}_{2}(\mathrm{BN} \times \mathrm{S})$ & $\begin{array}{c}S S \\
168.9 \pm 3.58(28)\end{array}$ & $\begin{array}{c}S B \\
176.4 \pm 3.38(43)\end{array}$ & $\begin{array}{c}B B \\
176.0 \pm 4.21(23)\end{array}$ & -7.1 & 0.30 \\
\hline $\mathrm{F}_{2}(\mathrm{~S} \times \mathrm{MNS}) *$ & $\begin{array}{c}S S \\
175.9 \pm 3.49(50)\end{array}$ & $\begin{array}{c}S M \\
171.7 \pm 2.19(74)\end{array}$ & $\begin{array}{c}M M \\
173.2 \pm 3.06(43)\end{array}$ & +2.7 & 0.56 \\
\hline
\end{tabular}

Blood pressure data are mean $\pm \mathrm{SE}$ in mmHg for each genotype. $S, \mathrm{~S}$ allele; $L, \mathrm{LEW}$ allele; $W, \mathrm{WKY}$ allele; $R, \mathrm{R}$ allele, $B, \mathrm{BN}$ allele; $M$, MNS allele; number of rats in each genotypic class are in parenthesis; "Blood pressure effect" is the difference in blood pressure of homozygous $S S$ minus blood pressure of homozygotes for the contrasting allele. *The genotype of the $\mathrm{F}_{2}(\mathrm{~S} \times \mathrm{MNS})$ population was done with $C Y P 2 B 2$ primers as there was no polymorphism between $\mathrm{S}$ and MNS using $C Y P 2 A 3$ primers. 
Table VI. Systolic Blood Pressure by Genotype for Selected Loci on Rat Chromosomes 1, 5, and 17 in the $F_{2}(L E W \times S)$

Population Raised on $8 \% \mathrm{NaCl}$ Diet

\begin{tabular}{|c|c|c|c|c|c|c|c|c|}
\hline \multirow[b]{2}{*}{ Locus } & \multirow[b]{2}{*}{ Chromosome } & \multicolumn{3}{|c|}{ Blood pressure by genotype } & \multirow[b]{2}{*}{ Blood pressure effect } & \multirow{2}{*}{$\begin{array}{c}\text { One-way ANOVA } \\
\text { probability }\end{array}$} & \multirow[b]{2}{*}{ DOM } & \multirow{2}{*}{$\begin{array}{l}\text { Percentage of } \\
\text { total variance }\end{array}$} \\
\hline & & SS & $S L$ & $L L$ & & & & \\
\hline$S A$ & $1 *$ & $200.7 \pm 5.18$ & $184.7 \pm 4.55$ & $176.7 \pm 3.20$ & +24.0 & 0.0045 & 0.48 & 7.3 \\
\hline$n$ & & 50 & 68 & 33 & & & & \\
\hline $\begin{array}{l}\text { D1Mco1 } \\
n\end{array}$ & $1 *$ & $\begin{array}{c}205.6 \pm 6.34 \\
38\end{array}$ & $\begin{array}{c}185.7 \pm 3.51 \\
78\end{array}$ & $\begin{array}{c}175.2 \pm 5.62 \\
34\end{array}$ & +30.4 & 0.0006 & 0.40 & 9.6 \\
\hline $\begin{array}{c}G L U T B^{\S} \\
n\end{array}$ & 5 & $\begin{array}{c}202.1 \pm 5.67 \\
38\end{array}$ & $\begin{array}{c}192.0 \pm 4.44 \\
72\end{array}$ & $\begin{array}{c}168.8 \pm 2.91 \\
41\end{array}$ & +33.3 & $<0.0001$ & 0.22 & 13.0 \\
\hline $\begin{array}{c}H I T H^{\S} \\
n\end{array}$ & 17 & $\begin{array}{c}187.3 \pm 4.61 \\
43\end{array}$ & $\begin{array}{c}195.9 \pm 4.26 \\
79\end{array}$ & $\begin{array}{c}168.9 \pm 4.73 \\
29 \\
\text { Sums: }\end{array}$ & $106.1 \mathrm{mmHg}$ & $\begin{array}{c}0.0015 \\
(0.0008)^{\ddagger}\end{array}$ & 0.002 & $38.3 \%$ \\
\hline
\end{tabular}

Blood pressure data is mean $\pm \mathrm{SE}$ in $\mathrm{mmHg}$ for each genotype. $S$, allele for $\mathrm{S}$ rats, $L$, allele for Lewis rats; "Blood pressure effect" is the difference in blood pressure of homozygous $S S$ minus blood pressure of homozygous $L L$ at each locus; DOM, probability associated with the test for dominance; $n$, number of rats in each genotypic class. See Table I for locus designations. * $S A$ and D1Mco1 loci are $55 \mathrm{cM}$ apart, see Figs. 1 or $2 .{ }^{*}$ Probability from one-way ANOVA assuming dominance of S allele. ${ }^{\S}$ Data were published previously (25) but are included here for the purpose of summarizing additive genetic effects.

many false positives will result if significance levels too high, i.e., $P<0.05$, are accepted. The QTL region marked by the $S A$ locus is supported by a LOD of only 2.4 and $P=0.0045$ by an ANOVA. Thus the evidence by itself is only suggestive of a QTL in this region. But since the present evidence serves to corroborate previously published data from three independent $\mathrm{F}_{2}$ populations derived from SHR or stroke prone-SHR and WKY indicating linkage of the $S A$ locus to blood pressure (6, $8,9)$, confidence that the result is correct is enhanced. It is interesting that the peak LOD score is essentially at the $S A$ gene. This does not, of course, establish the SA gene as the blood pressure QTL in this region. The $\beta$ and $\gamma$ subunits of the renal epithelial sodium channel are closely linked to the $S A$ locus on human chromosome 16 (32). Thus it is likely that these sodium channel subunits are closely linked to the SA locus on rat chromosome 1. Certain mutations in either the $\beta$ or $\gamma$ subunits cause activation of the sodium channel and result in a Mendelian form of inherited hypertension in humans (Liddles syndrome, pseudoaldosteronism) $(32,33)$. Clearly these genes are also major candidates for the blood pressure QTL in the SA region of rat chromosome 1.

The new putative QTL on chromosome 1 that is $55 \mathrm{cM}$ from the $S A$ region and marked by D1Mco1 has a somewhat better statistical support (LOD score of 3.4 and $P=0.0006$ by

Table VII. Heart Weight by Genotype for Selected Loci on Rat Chromosomes 1, 5, and 17 in the $F_{2}(L E W \times S)$ Population Raised on $8 \% \mathrm{NaCl}$ Diet

\begin{tabular}{|c|c|c|c|c|c|c|c|c|}
\hline \multirow[b]{2}{*}{ Locus } & \multirow[b]{2}{*}{ Chromosome } & \multicolumn{3}{|c|}{ Heart weight by genotype } & \multirow{2}{*}{$\begin{array}{l}\text { Heart weight } \\
\text { effect }\end{array}$} & \multirow{2}{*}{$\begin{array}{l}\text { One-way ANOVA } \\
\text { probability }\end{array}$} & \multirow[b]{2}{*}{ DOM } & \multirow{2}{*}{$\begin{array}{l}\text { Percentage of } \\
\text { total variance }\end{array}$} \\
\hline & & $S S$ & $S L$ & $L L$ & & & & \\
\hline $\begin{array}{r}S A \\
n\end{array}$ & $1^{*}$ & $\begin{array}{c}1,266 \pm 24.5 \\
50\end{array}$ & $\begin{array}{c}1,197 \pm 22.4 \\
68\end{array}$ & $\begin{array}{c}1,184 \pm 18.4 \\
33\end{array}$ & +82 & $\begin{array}{c}0.037 \\
(0.001)^{\ddagger}\end{array}$ & 0.30 & 4.4 \\
\hline $\begin{array}{l}\text { D1Mco1 } \\
n\end{array}$ & $1^{*}$ & $\begin{array}{c}1,288 \pm 34.2 \\
38\end{array}$ & $\begin{array}{c}1,211 \pm 16.4 \\
78\end{array}$ & $\begin{array}{c}1,157 \pm 24.8 \\
34\end{array}$ & +131 & 0.0036 & 0.67 & 7.3 \\
\hline $\begin{array}{c}G L U T B \\
n\end{array}$ & 5 & $\begin{array}{c}1,302 \pm 30.8 \\
38\end{array}$ & $\begin{array}{c}1,217 \pm 19.5 \\
72\end{array}$ & $\begin{array}{c}1,137 \pm 16.8 \\
41\end{array}$ & +165 & $<0.0001$ & 0.91 & 13.0 \\
\hline $\begin{array}{c}H I T H \\
n\end{array}$ & 17 & $\begin{array}{c}1,240 \pm 25.0 \\
43\end{array}$ & $\begin{array}{c}1,242 \pm 20.3 \\
79\end{array}$ & $\begin{array}{c}1,112 \pm 17.1 \\
29 \\
\text { Sums: }\end{array}$ & $\begin{array}{r}+128 \\
506 \mathrm{mg}\end{array}$ & $\begin{array}{c}0.0008 \\
(0.0002)^{\S}\end{array}$ & 0.009 & $\begin{array}{c}9.0 \\
33.7 \%\end{array}$ \\
\hline
\end{tabular}

Heart weight data is mean $\pm \mathrm{SE}$ in $\mathrm{mg}$ for each genotype. $S$, allele for $\mathrm{S}$ rats; $L$, allele for LEW rats; "Heart weight effect" is the difference in heart weight of homozygous $S S$ minus heart weight of homozygous $L L$ at each locus; DOM, probability associated with the test for dominance; $n=$ number of rats in each genotypic class. See Table I for locus designations. ${ }^{*} S A$ and D1Mco1 loci are $55 \mathrm{cM}$ apart, see Figs. 1 or $2 .{ }^{*}$ Probability for one-way ANOVA using body weight as a covariate. This corrects for an effect of the $S A$-locus chromosomal region on body weight. ${ }^{\circledR}$ Probability from one-way ANOVA assuming dominance of S allele. 
an ANOVA) than the $S A$ region. Candidate genes in this region include a cluster of cytochromes $\mathrm{P} 450$. Some cytochromes P450 are involved in the production of biologically active compounds from arachidonic acid. These metabolites have important effects on vascular smooth muscle and ion transport in kidney tubule cells (34-36). Although none of the cytochromes P450 on our present map are known to have this function, the possibility certainly exists that unknown cytochrome(s) P450 with this function exist as part of this gene cluster. The $\mathrm{Na}, \mathrm{K}$ ATPase $\alpha 3$ isoform (ATP1A3) is also a candidate locus very close to the cytochrome P450 gene cluster (Fig. 1). The cluster of kallikrein-like proteinases (marked by the $K A L$ locus) is also at the edge of this QTL region (compare Figs. 1 and 2).

The effect of the blood pressure QTL marked by the cytochromes P450 cluster on chromosome 1 was highly population dependent, being seen only in the $\mathrm{F}_{2}$ population derived from $\mathrm{S}$ and LEW strains, but not in $\mathrm{F}_{2}$ populations derived from $\mathrm{S}$ and other strains (Table V). This was true also of the $S A$ locus on chromosome 1 (Table IV). Cross specificity has invariably been the case with other QTL we have described on rat chromosomes 2 (30), $3(25,37) 5(25), 10(31,38), 13(14)$, and 17 (25). We attribute this to the fact that different alleles at each QTL can obviously be segregating in each cross depending on the strain crossed with $\mathrm{S}$ and to effects of genetic background also dependent on the strain crossed with S. The theoretical basis of this phenomenon is discussed in detail in a recent review (4).

In the $\mathrm{F}_{2}(\mathrm{LEW} \times \mathrm{S})$ population there was a strong correlation between heart weight and blood pressure. Since left ventricular hypertrophy is well known to occur with chronically increased blood pressure, the heart weight data are taken to corroborate and validate the blood pressure measurements.

The $\mathrm{F}_{2}(\mathrm{LEW} \times \mathrm{S})$ population is particularly favorable since we know of four QTL marked by the $S A$, D1Mco1, $G L U T B$, and HITH loci segregating in this population. These loci collectively accounted for $106 \mathrm{mmHg}$ of blood pressure and $506 \mathrm{mg}$ of heart wt, which was 38 and $34 \%$ of the total phenotypic variances, respectively. We do not have a measure of genetic variance for the $\mathrm{F}_{2}(\mathrm{LEW} \times \mathrm{S})$ population, so we cannot state the percentage of the genetic variance accounted for by the four known QTL. It is, however, obvious that a substantial part of the genetic variance is accounted for by these four loci.

Screening segregating populations for QTL is clearly only a first step in establishing the existence of a QTL in a chromosomal region. Because of the imprecision with which a QTL can be detected and localized it will be necessary to create congenic strains by substituting, for example, putative QTL chromosomal regions lowering blood pressure into the background of the S rats (4) and to demonstrate that such a strain actually has reduced blood pressure compared to S. In the case of chromosome 1, the two QTL proposed here should be tested by the construction of two such congenic strains, each containing nonoverlapping Lewis chromosome 1 regions, where each congenic strain is predicted to have lower blood pressure than the S strain.

\section{Acknowledgments}

This work was supported by grants from the National Institutes of Health and by the Helen and Harold McMaster Chair in Biochemistry and Molecular Biology.

\section{References}

1. Tanksley, S.D. 1993. Mapping polygenes. Annu. Rev. Genet. 27:205-233.

2. Lander, E.S., and M.J. Schork. 1994. Genetic dissection of complex traits. Science (Wash. DC). 265:2037-2048.

3. Collins, F.S. 1995. Positional cloning moves from perditional to traditional. Nat. Genet. 9:347-350.

4. Rapp, J.P., and A.Y. Deng. 1995. Detection and positional cloning of blood pressure quantitative trait loci: Is it possible? Hypertension (Dallas). 25: 1121-1128.

5. Iwai, N., and T. Inagami. 1991. Isolation of preferentially expressed genes in the kidneys of hypertensive rats. Hypertension (Dallas). 17:161-169.

6. Lindpaintner, K., P. Hilbert, D. Ganten, B. Nadal-Ginard, T. Inagami, and N. Iwai. 1993. Molecular genetics of the $\mathrm{S}_{\mathrm{A}}$-gene: cosegregation with hypertension and mapping to rat chromosome 1. J. Hypertens. 11:19-23.

7. Szpirer, C., M. Riviere, J. Szpirer, G. Levan, D.F. Guo, N. Iwai, and T. Inagami. 1993. Chromosomal assignment of human and rat hypertension candidate genes: type 1 angiotensin II receptor genes and the $\mathrm{S}_{\mathrm{A}}$ gene. J. Hypertens. 11:919-925.

8. Iwai, N., and T. Inagami. 1992. Identification of a candidate gene responsible for the high blood pressure of spontaneously hypertensive rats. J. Hypertens. 10:1155-1157.

9. Samani, N.T., D. Lodwick, M. Vincent, C. Dubay, M.A. Kaiser, M.P. Kelly, M. Lo, J. Harris, J. Sassard, M. Lathrop, and J.D. Swales. 1993. A gene differentially expressed in the kidney of the spontaneously hypertensive rat cosegregates with increased blood pressure. J. Clin. Invest. 92:1099-1103.

10. Harris, E.L., H. Dene, and J.P. Rapp. 1993. S A $_{\text {gene and blood pressure }}$ cosegregation using Dahl salt-sensitive rats. Am. J. Hypertens. 6:330-334.

11. Iwai, N., N. Ohmichi, K. Hanai, Y. Nakamura, and M. Kinoshita. 1994. Human SA gene locus as a candidate locus for essential hypertension. Hypertension (Dallas). 23:375-380.

12. Nabika, T., A. Bonnardeaux, M. James, C. Julier, X. Jeunemaitre, P. Corvol, M. Lathrop, and F. Soubrier. 1995. Evaluation of the SA locus in human hypertension. Hypertension (Dallas). 25:6-13.

13. Rapp, J.P., and H. Dene. 1985. Development and characteristics of inbred strains of Dahl salt-sensitive and salt-resistant rats. Hypertension (Dallas). 7:340-349.

14. Rapp, J.P., H. Dene, and A.Y. Deng. 1994. Seven renin alleles in rats and their effects on blood pressure. J. Hypertens. 12:349-355.

15. Buñag, R.D., and J. Butterfield. 1982. Tail-cuff blood pressure measurement without external preheating in awake rats. Hypertension (Dallas). 4:898903.

16. Blin, N., and D.W. Stafford. 1976. Isolation of high-molecular-weight DNA. Nucleic Acids Res. 3:2303-2308.

17. Hoebee, B., J.M. de Stoppelaar, R.F. Suijkerbuijk, and S. Monard. 1994. Isolation of rat chromosome-specific paint probes by bivariate flow sorting followed by degenerate oligonucleotide primed-PCR. Cytogenet. Cell Genet. 66: 277-282.

18. Telenius, H., A.H. Pelmear, A. Tunnacliffe, N.P. Carter, A. Behmel, M.A. Ferguson-Smith, M. Nordenskjöld, R. Pfragner, and B.A.J. Ponder. 1992. Cytogenetic analysis by chromosome painting using DOP-PCR amplified flowsorted chromosomes. Genes Chromosomes \& Cancer. 4:257-263.

19. Telenius, H., N.P. Carter, C. Bebb, M. Nordenskjöld, B.A.J. Ponder, and A. Tunnacliffe. 1992. Degenerate oligonucleotide-primed PCR: general amplification of target DNA by a single degenerate primer. Genomics. 13:718725 .

20. Serikawa, T., T. Kuramoto, P. Hilbert, M. Mori, J. Yamada, C.J. Dubay, K. Lindpainter, D. Ganten, J.-L. Guénet, G.M. Lathrop, and J.S. Beckmann. 1992. Rat gene mapping using PCR-analyzed microsatellites. Genetics. 131:701721.

21. Jacob, H.J., D.M. Brown, R.K. Bunker, M.J. Daly, V.J. Dzau, A. Goodman, G. Koike, V. Kren, T. Kurtz, A. Lernmark, et al. 1995. A genetic linkage map of the laboratory rat, Rattus norvegicus. Nat. Genet. 9:63-69.

22. Kunieda, T., E. Kobayashi, M. Tachibana, H. Ikadai, and T. Imamichi. 1992. Polymorphic microsatellite loci of the rat (Rattus norvegicus). Mamm. Genome. 3:564-567.

23. Goldmuntz, E.A., E.F. Remmers, H. Zha, J.M. Cash, P. Mathern, L.J. Crofford, and R.L. Wilder. 1993. Genetic map of 12 polymorphic loci on rat chromosome 1. Genomics. 16:761-764.

24. Gu, L., H. Dene, and J.P. Rapp. 1994. Three microsatellites defining four alleles for the rat SA gene. Mamm. Genome. 5:833.

25. Deng, A.Y., H. Dene, M. Pravenec, and J.P. Rapp. 1994. Genetic mapping of two new blood pressure quantitative trait loci in the rat by genotyping endothelin system genes. J. Clin. Invest. 93:2701-2709.

26. Lander, E., P. Green, J. Abrahamson, A. Barlow, M.J. Daly, S.E. Lincoln, and L. Newburg. 1987. MAPMAKER: an interactive computer package for constructing primary genetic linkage maps of experimental and natural populations. Genomics. 1:174-181.

27. Lander, E., and D. Bostein. 1989. Mapping Mendelian factors underlying quantitative traits using RFLP linkage maps. Genetics. 12:185-199.

28. Darvasi, A., A. Weinreb, V. Minke, J.I. Weller, and M. Soller. 1993. Detecting marker-QTL linkage and estimating QTL gene effect and map location 
using a saturated genetic map. Genetics. 134:943-951.

29. Martinez, O., and R.N. Curnow. 1992. Estimating the locations and the sizes of the effects of quantitative trait loci using flanking markers. Theor. Appl. Genet. 85:480-488.

30. Deng, A.Y., H. Dene, and J.P. Rapp. 1994. Mapping of a quantitative trait locus for blood pressure on rat chromosome 2. J. Clin. Invest. 94:431-436.

31. Deng, A.Y., and J.P. Rapp. 1995. Locus for the inducible, but not a constitutive, nitric oxide synthase cosegregates with blood pressure in the Dahl salt-sensitive rat. J. Clin. Invest. 95:2170-2177.

32. Hansson, J.H., C. Nelson-Williams, H. Suzuki, L. Schild, R. Shimkets, Y. Lu, C. Canessa, T. Iwasaki, B. Rossier, and R.P. Lifton. 1995. Hypertension caused by a truncated epithelial sodium channel $\gamma$ subunit: genetic heterogeneity of Liddle syndrome. Nat. Genet. 11:76-82.

33. Shimkets, R.A., D.G. Warnock, C.M. Bositis, C. Nelson-Williams, J.H. Hansson, M. Schambelan, J.R. Gill, Jr., S. Ulick, R.V. Milora, J.W. Findling, et al. 1994. Liddle's syndrome: heritable human hypertension caused by mutations in the $\beta$ subunit of the epithelial sodium channel. Cell. 79:407-414.

34. McGiff, J.C. 1991. Cytochrome P-450 metabolism of arachidonic acid. Annu. Rev. Pharmacol. Toxicol. 31:339-369.

35. McGiff, J.C., M.A. Carroll, and B. Escalante. 1991. Arachidonate metabolites and kinins in blood pressure regulation. Hypertension (Dallas). 18(suppl. III):III-150-III-157.

36. Hirt, D.L., and H.R. Jacobson. 1991. Functional effects of cytochrome P450 arachidonate metabolites in the kidney. Seminars in Nephrology. 11:148155 .

37. Cicila, G.T., J.P. Rapp, K.D. Bloch, T.W. Kurtz, M. Pravenec, V. Křen, C.C Hong, T. Quertermous, and S.C. Ng. 1994. Cosegregation of the endothelin-3 locus with blood pressure and relative heart weight in inbred Dahl rats. $J$. Hypertens. 12:643-651.

38. Deng, Y., and J.P. Rapp. 1992. Cosegregation of blood pressure with angiotensin converting enzyme and atrial natriuretic peptide receptor genes using Dahl salt-sensitive rats. Nat. Genet. 1:267-272. 